\title{
Kinematic design of a non-parasitic 2R1T parallel mechanism with remote center of motion to be used in minimally invasive surgery applications
}

\author{
Abdullah Yaşır ${ }^{\mathrm{a}}$, Gökhan Kiper ${ }^{\mathrm{a}, \mathrm{b}, *}$, M.I. Can Dede ${ }^{\mathrm{b}}$ \\ a Precision and Microsystems Engineering Department, Delft University of Technology, 2628 CD Delft, Netherlands \\ ${ }^{\mathrm{b}}$ Mechanical Engineering Department, Izmir Institute of Technology, 35430 Izmir, Turkey
}

\section{A R T I C L E I N F O}

\section{Article history:}

Received 20 March 2020

Revised 24 May 2020

Accepted 29 June 2020

Available online 6 July 2020

\section{Keywords:}

Surgical robotics

Parallel manipulator

2R1T mechanism

Remote center of motion

\begin{abstract}
A B S T R A C T
In minimally invasive surgery applications, the use of robotic manipulators is becoming more and more common to enhance the precision of the operations and post-operative processes. Such operations are often performed through an incision port (a pivot point) on the patient's body. Since the end-effector (the handled surgical tool) move about the pivot point, the manipulator has to move about a remote center of motion. In this study, a 3-degrees-of-freedom parallel mechanism with 2R1T (R: rotation, T: translation) remote center of motion capability is presented for minimally invasive surgery applications. First, its kinematic structure is introduced. Then, its kinematic analysis is carried out by using a simplified kinematic model which consists of three intersecting planes. Then the dimensional design is done for the desired workspace and a simulation test is carried out to verify the kinematic formulations. Finally, the prototype of the final design is presented.
\end{abstract}

(c) 2020 Elsevier Ltd. All rights reserved.

\section{Introduction}

Use of robots in the medical field started by adapting industrial robots to surgical applications $[3,8]$. These industrial robots were used after some modifications to guarantee surgical requirements such as safety and sterility. Firstly in 1985, Kwoh et al. [8] used a PUMA 560 industrial robot to locate a neurosurgical tool next to the head of the patient. Recently robots began to take place in surgical rooms as assistive devices for the surgeons [6]. However, industrial robots and surgical robots differ from each other in their priorities. Taniguchi et al. [12] state that an industrial robot's primary characteristics are "high power" and "high speed" while a surgical robot's are "safety" and "cleanness".

Due to the specific requirements of surgical applications, highly specialized systems were needed over time [13] and surgical robots have been developed to satisfy these requirements. Minimally invasive surgery (MIS) is one of these applications in which specifically designed surgical robots are commonly used. MIS is performed with surgical tools or instruments inserted through a small hole on the patient's body. This hole is also known as incision port, fulcrum, trocar or pivot point depending on the MIS application. Surgeons and patients prefer MIS since these operations can be completed in reduced durations with minimal pain and blood loss, and lower risk of infection. Post-operative processes also result in faster recovery and smaller surgical scars [9].

\footnotetext{
* Corresponding author: Professor Gökhan Kiper, Izmir Institute of Technology, Izmir Institute of Technology, Department of Mechanical Engineering, 35430 İzmir, Turkey, Tel.: +90 232 7506777; Fax: +90 2327506701.

E-mail address: gokhankiper@iyte.edu.tr (G. Kiper).
} 


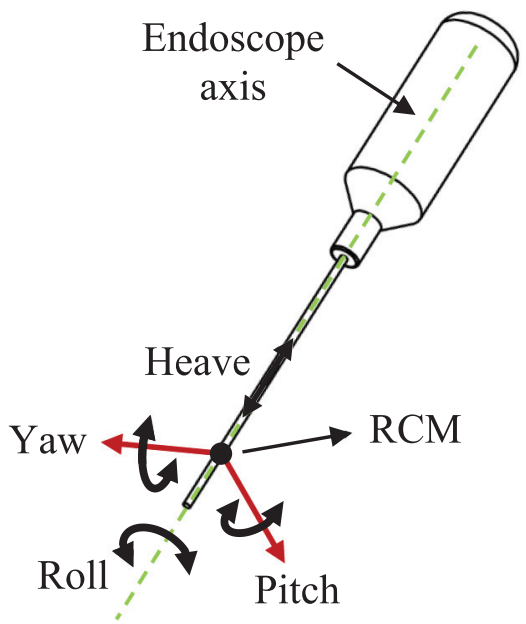

Fig. 1. Description of the motions of an endoscope with a remote center of motion.

Taniguchi et al. [12] state that 27 endoscope robots have been developed between 1994 and 2009 and 8 of them have been used on humans while others have been used on animals or stayed as a model. In most of these systems, the endoscope is positioned with a robotic arm and the surgeon controls this robotic arm with different tools or ways such as using a controller, giving voice commands, making head movements or using an image processing system tracking the surgical tools in the surgeon's hand. Bihlmaier [2] explains the main examples of the motorized endoscope holding robots in literature in chronological order of development and states that most of them are designed for laparoscopic surgery applications and have serial structures with various degrees-of-freedoms (dof).

The kinematic design stage is one of the most important stages in designing an MIS robot since some crucial MIS concerns such as safety, accuracy, dexterity and ergonomics are considered at this phase. Therefore, kinematic design considerations such as pivoting motion, decoupled motion, back-drivability, redundancy, workspace and isotropy should be taken into account to fulfil specific surgical requirements [7]. In this regard, pivoting motion through a remote center of motion (RCM) is required due to the necessity of moving a tool or camera through/about an incision port in MIS. This requirement can be satisfied in two different ways: by using a mechanical RCM or a non-mechanical RCM. The first way is to force the surgical tool mechanically to move around a center of motion that is outside the robot's structure. RCM is a point where one or more rotational and translational movements are pivoted outside the mechanism. This concept allows MIS robots to work in a larger space without touching the body of the patient while providing the desired pivoting movements of the surgical tool. In addition, while the robot is operating, the patient and surgeon are automatically protected against the damages caused by any possible control or coordination error [6]. In robotic MIS systems, mechanical RCMs can be obtained by using several concepts: isocenters, circular tracking arcs, parallelograms, synchronous belt transmissions, spherical linkages, parallel mechanisms and gear trains [7]. The second way is to use a non-mechanical RCM concept, where the desired pivoted motion is obtained at a virtual RCM by the control of a redundant robot. However, Liu et al. [11] state that compared to non-mechanical ones, mechanical RCMs are more reliable and considered more suitable for clinical practice.

In MIS, a surgical tool or instrument may have up to 4 ( 3 rotational +1 translational) dof through the incision port: pitch, yaw, roll and heave motions (Fig. 1). For endoscope holding robots, pitch, yaw and heave motions are sufficient for endoscope movements and the roll motion about the endoscope axis is usually unnecessary. These three motions define a 2R1T (R: rotation; T: translation) motion pattern where the translation direction is perpendicular to the plane defined by the orthogonally intersecting rotation axes. In this case, the intersection point of the rotation axes is the RCM.

Although there are several patents and papers on serial and hybrid manipulators with 2R1T motion, parallel manipulators with 2R1T motion are not common. 2R1T parallel manipulators with a RCM presented by Li et al. [10] seem to have 3-URRR and 3-UPRR kinematic structures with all three legs equally spaced about the end-effector axis, but no details are given other than some figures. Nevertheless, the 3-URRR manipulator seems to have a similar architecture with the manipulator presented in this study. Recently Yaşır and Kiper [14] have presented the structural synthesis of 2R1T manipulators with RCM where all serial, hybrid and parallel architectures are considered. As a result of that study, the manipulator architecture used in this study is selected for the detailed design.

This study deals with the design of a parallel manipulator for an endoscope holding robot to be used in minimally invasive transnasal surgeries. The robot is an assistive robot to work alongside the surgeon during the surgery. Hence, this work is about designing a non-parasitic surgical robotic arm with a RCM which is capable of performing 2R1T motion such that an endoscope can be precisely manipulated without affecting the access of the surgeon to the operation area. The paper is organized as follows: In Section 2, the kinematic structure of the manipulator is explained and then it is simplified by 


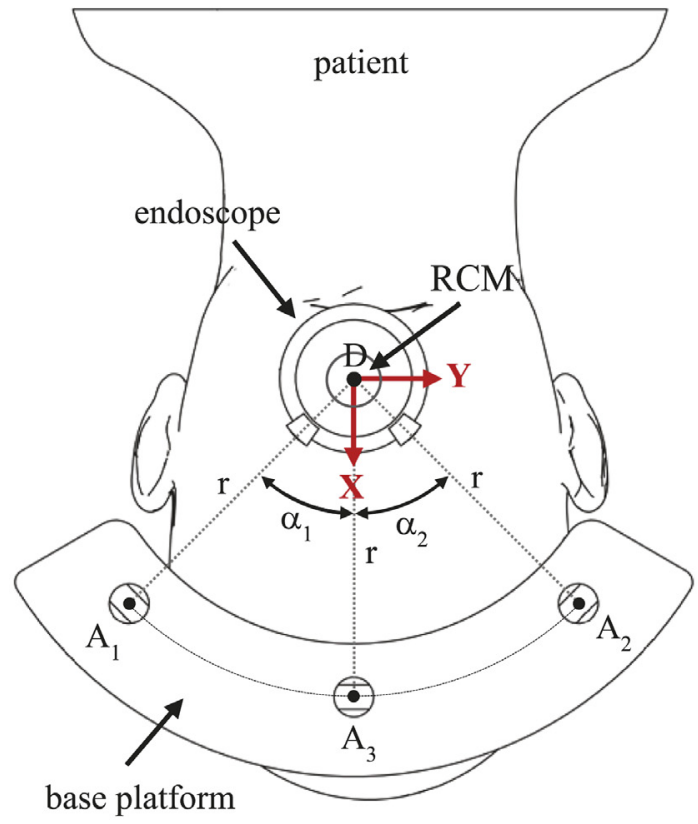

a)

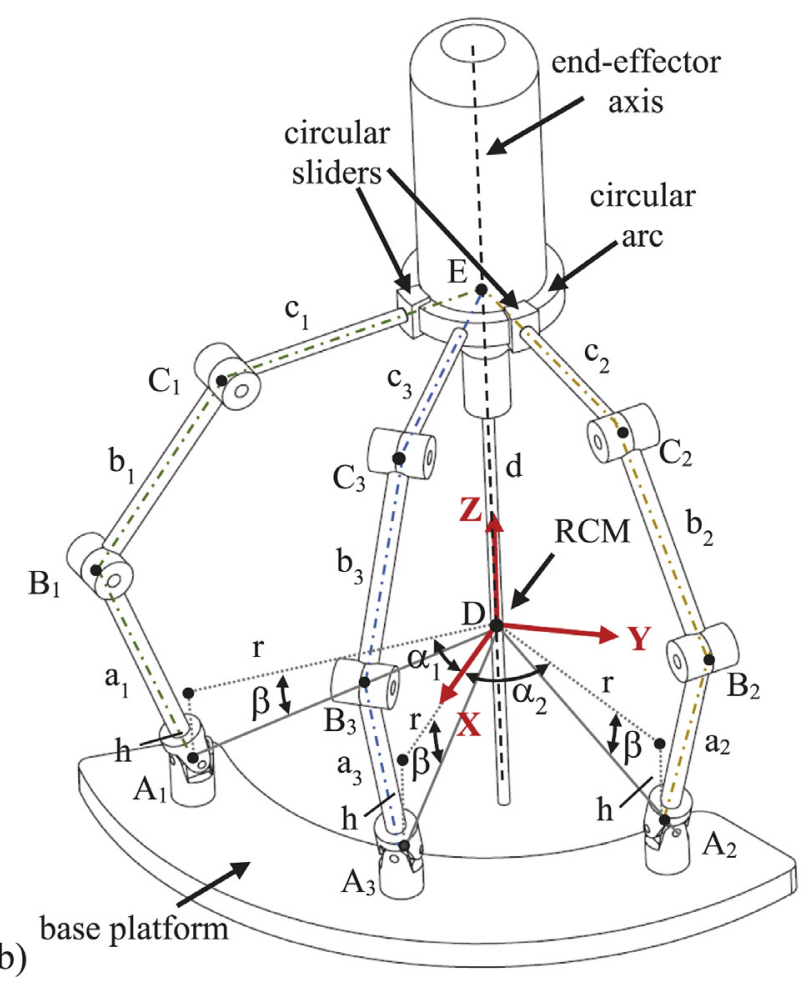

Fig. 2. Description of 2URRR-URR manipulator a) Top view on the patient, b) An auxiliary view.

using intersecting planes. Kinematic analysis and design of the parallel manipulator are presented in Section 3, where a slider-crank mechanism is designed for the middle leg of the manipulator and the link dimensions are optimized for the desired workspace. A Matlab simulation is presented in Section 4 to verify the direct and inverse kinematics formulations presented in Section 3. The constructional design and a prototype are presented in Section 5. Finally, Section 6 concludes the paper.

\section{Description of the manipulator}

A 3-dof parallel manipulator with 2R1T motion pattern and RCM is selected among several other alternatives [14]. The kinematic structure of the manipulator is 2URRR-URR which comprises 3 legs. The $U$ joints of those legs can be arbitrarily distributed on the base platform of the manipulator. As it can be observed from Fig. 2a, the legs are placed on one side on the base platform instead of distributing them symmetrically around the Z-axis. This is for the sake of providing the surgeon with some robot link free space to facilitate the access of the surgeon to the surgical area. In Fig. $2 \mathrm{~b}$, the kinematic diagram of the 2URRR-URR manipulator is given. As can be seen, the axes of the three $\mathrm{R}$ joints on the base platform intersect at the RCM point. The URR leg is selected as the middle leg and it is placed by rotating the first revolute joint axis by an angle $\beta$ from X-axis around Y-axis. The two URRR legs are selected as the side legs and they are placed by first rotating their first revolute joint axes by an angle $\alpha_{1}$ and $\alpha_{2}$ from X-axis to both sides around Z-axis, then rotating them by an angle $\beta$ around $\mathrm{Y}$-axis. The distance between the center of each $\mathrm{U}$ joint and the projection of the RCM point on the XY-plane is shown by $\mathrm{r}$ and the distances between these centers and XY-plane are represented by $h$. The last $\mathrm{R}$ joints of the two URRR legs are constructed by using circular sliders which slide on an arc attached to the endoscope group.

Except for the first $\mathrm{R}$ joints of all legs and the $\mathrm{R}$ joints of URRR legs about the end-effector axis at $\mathrm{E}$, all legs have three parallel revolute joints which define the planes $A_{1} B_{1} C_{1}, A_{2} B_{2} C_{2}$ and $A_{3} B_{3} C_{3}$ (Fig. 3). It means that each leg can move on a plane and the angle of this plane can be changed by a revolute joint whose axis is within that plane. Hence, a kinematic diagram can be represented by a simpler model which consists of three planes intersecting along the end-effector axis and the angle of each plane can be changed. As can be seen in Fig. 3, there are three planes and each of them represents an RRR leg. These planes are intersecting along $\vec{w}$ - unit vector along the end-effector axis. The angles of the side planes are $\theta_{1}$ and $\theta_{2}$, which are also the joint variables associated with the $\mathrm{R}$ joints between the base platform and the side legs. Once $\theta_{1}$ and $\theta_{2}$ are defined, the angle of the middle plane is already determined because they all have to include $\vec{w}$. For the middle leg, the second $\mathrm{R}$ joint can be used to manipulate the distance $|\mathrm{DE}|=d$. This joint angle is denoted as $\theta_{3}$. Thus, $\theta_{1}, \theta_{2}$ and $\theta_{3}$ are the input variables for the manipulator. Also, the unit normal vector of each plane is shown as $\vec{n}_{1}, \vec{n}_{2}, \vec{n}_{3}$, respectively. $\hat{X}(\cdot), \hat{Y}(\cdot)$ and $\hat{Z}(\cdot)$ represent the elementary rotation matrices about X-, Y- and Z-axes, respectively. 


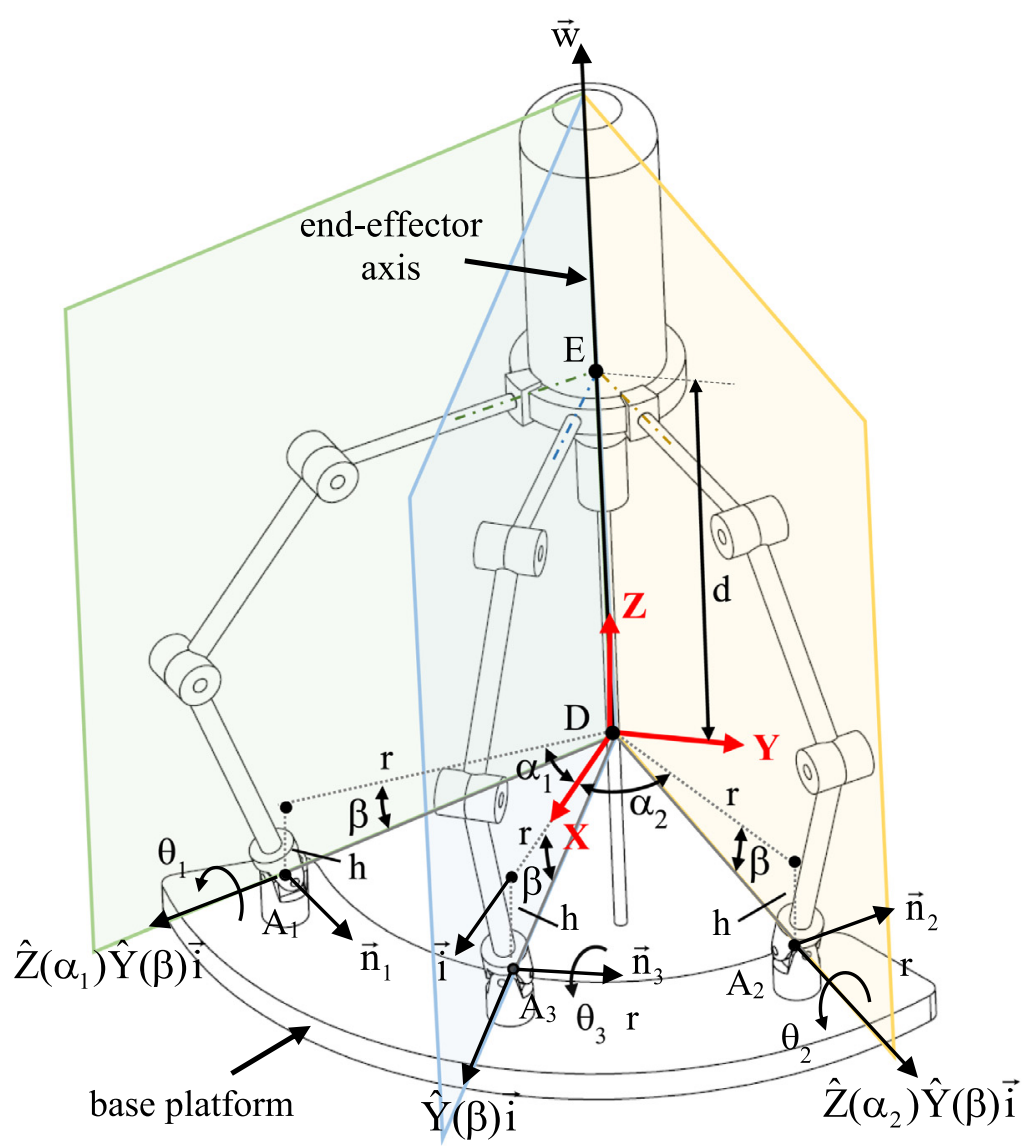

Fig. 3. Simplified kinematic diagram of the manipulator with three intersecting planes.

The end-effector axis remains at the intersection line of the three leg planes which always pass through the RCM, but can be oriented as desired. Due to this architecture of the manipulator, the end-effector axis can only have a variable orientation ( $2 \mathrm{R}$ motion) about the RCM and can slide through (1T motion) the RCM. Therefore, there is no parasitic motion of the endeffector. Details of the structural design of non-parasitic manipulators with RCM can be found in [10] and [14]. With the intersecting planes concept, direct and inverse kinematics of the mechanism can be easily formulated. This also facilitates the controller design for the robot.

\section{Kinematic formulation and design}

In this section, first the kinematic formulation to be used for the kinematic analysis and design of the manipulator is presented. Then, the direct and inverse kinematic analyses for position and velocity levels are presented. Finally, the kinematic design studies are presented.

\subsection{Kinematic formulation}

It should be noted that from this point on, abbreviations for cosine and sine functions are used as "c" and "s" wherever appropriate. Let $\phi$ be the angle of $\vec{w}$ with respect to the XZ-plane (corresponding to the yaw motion) and $\psi$ be the angle of $\vec{w}$ with respect to the YZ-plane (corresponding to the pitch motion). Then, $\vec{n}_{1}, \vec{n}_{2}$ and $\vec{w}$ vectors can be found as follows:

$$
\begin{aligned}
\vec{n}_{1} & =\hat{Z}\left(\alpha_{1}\right) \hat{Y}(\beta) \hat{X}\left(\theta_{1}\right)\left[\begin{array}{l}
0 \\
1 \\
0
\end{array}\right]=\left[\begin{array}{ccc}
c \alpha_{1} & -s \alpha_{1} & 0 \\
s \alpha_{1} & c \alpha_{1} & 0 \\
0 & 0 & 1
\end{array}\right]\left[\begin{array}{ccc}
c \beta & 0 & s \beta \\
0 & 1 & 0 \\
-s \beta & 0 & c \beta
\end{array}\right]\left[\begin{array}{ccc}
1 & 0 & 0 \\
0 & c \theta_{1} & -s \theta_{1} \\
0 & s \theta_{1} & c \theta_{1}
\end{array}\right]\left[\begin{array}{l}
0 \\
1 \\
0
\end{array}\right] \\
& =\left[\begin{array}{c}
c \alpha_{1} s \beta s \theta_{1}-s \alpha_{1} c \theta_{1} \\
s \alpha_{1} s \beta s \theta_{1}+c \alpha_{1} c \theta_{1} \\
c \beta s \theta_{1}
\end{array}\right]
\end{aligned}
$$




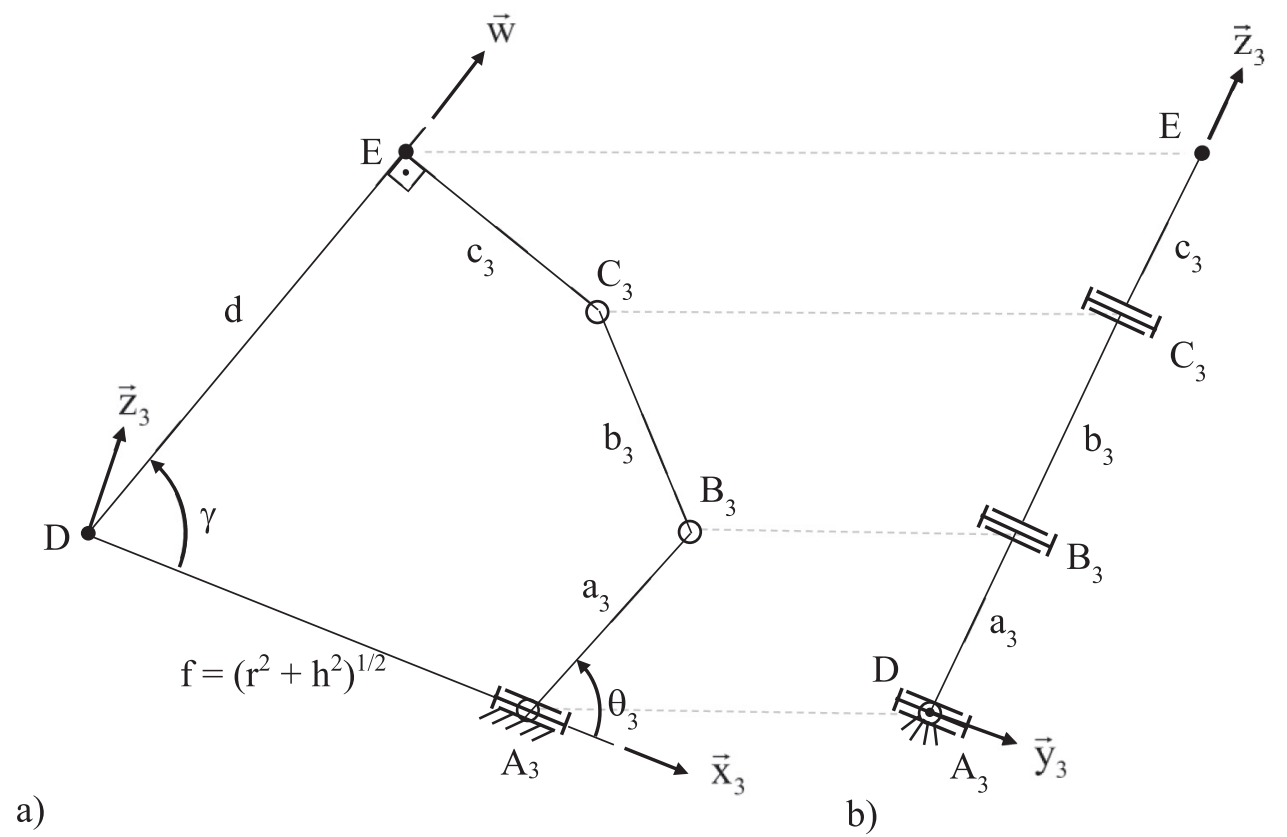

Fig. 4. Kinematic diagrams for the middle leg: a) on $\vec{x}_{3} \vec{z}_{3}-$ plane, b) on $\overrightarrow{y_{3}} \vec{z}_{3}-$ plane.

$$
\begin{aligned}
& \vec{n}_{2}=\hat{Z}\left(\alpha_{2}\right) \hat{Y}(\beta) \hat{X}\left(\theta_{2}\right)\left[\begin{array}{l}
0 \\
1 \\
0
\end{array}\right]=\left[\begin{array}{c}
c \alpha_{2} s \beta s \theta_{2}-s \alpha_{2} c \theta_{2} \\
s \alpha_{2} s s \theta_{2}+c \alpha_{2} c \theta_{2} \\
c \beta s \theta_{2}
\end{array}\right] \\
& \vec{w}=\left[\begin{array}{c}
s \psi \\
-s \phi \\
\sqrt{1-s^{2} \phi-s^{2} \psi}
\end{array}\right]=\frac{\vec{n}_{1} \times \vec{n}_{2}}{\left|\vec{n}_{1} \times \vec{n}_{2}\right|}
\end{aligned}
$$

where

$$
\begin{aligned}
\vec{n}_{1} \times \vec{n}_{2} & =\left[\begin{array}{ccc}
\vec{i} & \vec{j} & \vec{k} \\
c \alpha_{1} s \beta s \theta_{1}-s \alpha_{1} c \theta_{1} & s \alpha_{1} s \beta s \theta_{1}+c \alpha_{1} c \theta_{1} & c \beta s \theta_{1} \\
c \alpha_{2} s \beta s \theta_{2}-s \alpha_{2} c \theta_{2} & s \alpha_{2} s \beta s \theta_{2}+c \alpha_{2} c \theta_{2} & c \beta s \theta_{2}
\end{array} \mid\right. \\
& =\left[\begin{array}{c}
\left(s \alpha_{1}-s \alpha_{2}\right) c \beta s \beta s \theta_{1} s \theta_{2}+c \alpha_{1} c \beta c \theta_{1} s \theta_{2}-c \alpha_{2} c \beta s \theta_{1} c \theta_{2} \\
\left(c \alpha_{2}-c \alpha_{1}\right) c \beta s \beta s \theta_{1} s \theta_{2}-s \alpha_{2} c \beta s \theta_{1} c \theta_{2}+s \alpha_{1} c \beta c \theta_{1} s \theta_{2} \\
s\left(\alpha_{2}-\alpha_{1}\right)\left(c \theta_{1} c \theta_{2}+s^{2} \beta s \theta_{1} s \theta_{2}\right)+c\left(\alpha_{2}-\alpha_{1}\right) s \beta s\left(\theta_{1}-\theta_{2}\right)
\end{array}\right]
\end{aligned}
$$

For symmetrical positioning of the side legs, $\alpha_{1}=-\alpha$ and $\alpha_{2}=\alpha$. Then,

$$
\begin{aligned}
& \vec{n}_{1} \times \vec{n}_{2}=\left[\begin{array}{c}
-s \alpha s 2 \beta s \theta_{1} s \theta_{2}+c \alpha c \beta s\left(\theta_{2}-\theta_{1}\right) \\
-s \alpha c \beta s\left(\theta_{1}+\theta_{2}\right) \\
s 2 \alpha\left(c \theta_{1} c \theta_{2}+s^{2} \beta s \theta_{1} s \theta_{2}\right)+c 2 \alpha s \beta s\left(\theta_{1}-\theta_{2}\right)
\end{array}\right] \\
& \Rightarrow\left|\vec{n}_{1} \times \vec{n}_{2}\right|=\sqrt{\begin{array}{c}
\left(-\operatorname{s} \alpha s 2 \beta s \theta_{1} s \theta_{2}+\operatorname{coc} \beta s\left(\theta_{2}-\theta_{1}\right)\right)^{2}+\left(\operatorname{sic} \beta s\left(\theta_{1}+\theta_{2}\right)\right)^{2} \\
+\left(\operatorname{s} 2 \alpha\left(c \theta_{1} c \theta_{2}+s^{2} \beta s \theta_{1} s \theta_{2}\right)+\operatorname{c} 2 \alpha s \beta s\left(\theta_{1}-\theta_{2}\right)\right)^{2}
\end{array}}
\end{aligned}
$$

In Fig. 4.a, base segment $D_{3}$, leg chain $A_{3} B_{3} C_{3}$, platform segment $C_{3} E$ and end-effector line ED are coplanar. $D A_{3}$ direction is obtained by rotating $\mathrm{X}$-axis about Y-axis by angle $\beta$. So, the unit vector along $\mathrm{DA}_{3}$ is $\vec{x}_{3}=[\mathrm{c} \beta 0-\mathrm{s} \beta]^{\mathrm{T}}$. Unit vector $\vec{w}$ along DE is already known. So, $\mathrm{A}_{3} \mathrm{DE}$ angle $\gamma$ can be found as:

$$
\gamma=\cos ^{-1}\left(\vec{w} \cdot \vec{x}_{3}\right)=\cos ^{-1}\left(c \beta s \psi-s \beta \sqrt{1-s^{2} \phi-s^{2} \psi}\right)
$$

In local coordinates, $\vec{x}_{3}$ is located on the horizontal plane. So, locations of $C_{3}$ and $B_{3}$ are $\left(d--i c_{3}\right) e^{i \gamma}$ and $f+a_{3} e^{i \theta_{3}}$, respectively. Then,

$$
\left|\left(d-i c_{3}\right) e^{i \gamma}-f-a_{3} e^{i \theta_{3}}\right|=b_{3}
$$




\subsubsection{Direct kinematics}

For direct kinematics, by using Eqs. (5) and (6), the end-effector orientation angles $\psi$ and $\phi$ are found as:

$$
\begin{aligned}
& \psi=\sin ^{-1} \frac{-s \alpha s 2 \beta s \theta_{1} s \theta_{2}+\operatorname{coc} \beta s\left(\theta_{2}-\theta_{1}\right)}{\left|\vec{n}_{1} \times \vec{n}_{2}\right|} \\
& \phi=\sin ^{-1} \frac{s \alpha c \beta s\left(\theta_{1}+\theta_{2}\right)}{\left|\vec{n}_{1} \times \vec{n}_{2}\right|}
\end{aligned}
$$

If all the terms at the left-hand side of Eq. (8) are divided by $e^{i \gamma}$, the end-effector heave variable $\mathrm{d}$ can be found with the following steps:

$$
\begin{gathered}
\left|d-\left[i c_{3}+f e^{-i \gamma}+a_{3} e^{i\left(\theta_{3}-\gamma\right)}\right]\right|=b_{3} \Rightarrow\left(d-\left[i c_{3}+f e^{-i \gamma}+a_{3} e^{i\left(\theta_{3}-\gamma\right)}\right]\right)\left(d-\left[-i c_{3}+f e^{i \gamma}+a_{3} e^{-i\left(\theta_{3}-\gamma\right)}\right]\right)=b_{3}{ }^{2} \\
d^{2}-2 d\left[f c \gamma+a_{3} c\left(\theta_{3}-\gamma\right)\right]+\left[f c \gamma+a_{3} c\left(\theta_{3}-\gamma\right)\right]^{2}+\left[c_{3}-f s \gamma+a_{3} s\left(\theta_{3}-\gamma\right)\right]^{2}-b_{3}{ }^{2}=0 \\
\Rightarrow d=f c \gamma+a_{3} c\left(\theta_{3}-\gamma\right)+\sqrt{{b_{3}}^{2}-\left[c_{3}-f s \gamma+a_{3} s\left(\theta_{3}-\gamma\right)\right]^{2}}
\end{gathered}
$$

In Eq. (11), when the sign in front of the square root is selected as positive, this corresponds to the assembly mode shown in Fig. 4. When the sign is negative, line $C_{3} E$ is in a mirror-symmetrical position with respect to a line passing through $B_{3}$ and parallel to $C_{3} E$. This assembly mode corresponding to the negative side is unfavorable, because in this mode the links of the manipulator will be too much close to the patient's head. The singular configuration where $B_{3}, C_{3}$ and $E$ are collinear corresponds to the configuration where the transmission angle of the slider-crank linkage is zero, and as it is explained in Section 3.2.2, we designed the mechanism not to go near this configuration. Hence the slider-crank stays in the assembly mode shown in Fig. 4.

\subsubsection{Inverse kinematics}

For the inverse kinematics, since $\vec{w}$ and $\vec{n}_{1}$ are orthogonal vectors, $\vec{w} \cdot \vec{n}_{1}=0$. Using Eqs. (1) and (3),

$$
\begin{gathered}
\vec{w} \cdot \vec{n}_{1}=\left[\begin{array}{c}
s \psi \\
-s \phi \\
\sqrt{1-s^{2} \phi-s^{2} \psi}
\end{array}\right] \cdot\left[\begin{array}{c}
c \alpha s \beta s \theta_{1}+s \alpha c \theta_{1} \\
-s \alpha s \beta s \theta_{1}+c \alpha c \theta_{1} \\
c \beta s \theta_{1}
\end{array}\right]=0 \\
\Rightarrow(s \psi s \alpha-s \phi c \alpha) c \theta_{1}+\left[(s \psi c \alpha+s \phi s \alpha) s \beta+\sqrt{1-s^{2} \phi-s^{2} \psi} c \beta\right] s \theta_{1}=0
\end{gathered}
$$

Consequently, the left leg actuated variable $\theta_{1}$ is found by solving Eq. (12) as

$$
\theta_{1}=\tan ^{-1} \frac{s \phi c \alpha-\mathrm{s} \psi s \alpha}{(\mathrm{s} \psi c \alpha+s \phi s \alpha) s \beta+\sqrt{1-s^{2} \phi-s^{2} \psi} c \beta}
$$

Following similar procedure with $\vec{w} \cdot \vec{n}_{2}=0$, the right leg actuated variable $\theta_{2}$ is found as

$$
\theta_{2}=\tan ^{-1} \frac{s \phi c \alpha+\mathrm{s} \psi s \alpha}{(\mathrm{s} \psi c \alpha-s \phi s \alpha) s \beta+\sqrt{1-s^{2} \phi-s^{2} \psi} c \beta}
$$

When both sides of Eq. (8) are squared, it yields,

$$
\begin{gathered}
\left(d c \gamma+c_{3} s \gamma-f-a_{3} c \theta_{3}\right)^{2}+\left(d s \gamma-c_{3} c \gamma-a_{3} s \theta_{3}\right)^{2}=b_{3}{ }^{2} \\
2 a_{3}\left(d c \gamma+c_{3} s \gamma-f\right) c \theta_{3}+2 a_{3}\left(d s \gamma-c_{3} c \gamma\right) s \theta_{3}=\left(d c \gamma+c_{3} s \gamma-f\right)^{2}+\left(d s \gamma-c_{3} c \gamma\right)^{2}+a_{3}{ }^{2}-b_{3}{ }^{2} \\
\Rightarrow A c \theta_{3}+B s \theta_{3}=C
\end{gathered}
$$

where $A=2 a_{3}\left(d c \gamma+c_{3} s \gamma-f\right), B=2 a_{3}\left(d s \gamma-c_{3} c \gamma\right)$ and $C=\left(d c \gamma+c_{3} s \gamma-f\right)^{2}+\left(d s \gamma-c_{3} c \gamma\right)^{2}+a_{3}{ }^{2}-b_{3}{ }^{2}$. Let $A=$ Mc $\delta$, $B=$ Ms $\delta$ such that $M=\sqrt{A^{2}+B^{2}}=\left|\overrightarrow{A_{0} A_{2}}\right|$ and $\delta=\operatorname{atan} 2(A, B)=\measuredangle \vec{A}_{3} C_{3}$. Then, the middle leg actuated variable, $\theta_{3}$ is found as:

$$
A c \theta_{3}+B s \theta_{3}=\operatorname{Mc}\left(\theta_{3}-\delta\right)=C \Rightarrow \theta_{3}=\delta-\cos ^{-1}(\mathrm{C} / \mathrm{M})
$$

$\theta_{3}=\delta+\cos ^{-1}(\mathrm{C} / \mathrm{M})$ corresponds to the configuration shown in Fig. 4 , where joint $\mathrm{B}_{3}$ is to the right of line $\mathrm{A}_{3} \mathrm{C}_{3}$. $\theta_{3}=$ $\delta+\cos ^{-1}(\mathrm{C} / \mathrm{M})$ also satisfies Eq. (16), but this assembly mode corresponds to the configuration where $\mathrm{B}_{3}$ is to the left of line $A_{3} C_{3}$. Such an assembly mode is not favorable, because joint $B_{3}$ would come closer to the patient's head and $A_{3} B_{3}$ link may collide with the base link. For the selected workspace and selected link lengths in Section 3.2.2, the crank and coupler links of the slider-crank linkage never become collinear and assembly mode change does not occur. 
3.1.3. Velocity level kinematics

From Eqs. (3) and (5),

$$
\vec{w}=\left[\begin{array}{c}
s \psi \\
-s \phi \\
\sqrt{1-s^{2} \phi-s^{2} \psi}
\end{array}\right]=\frac{1}{\left|\vec{n}_{1} \times \vec{n}_{2}\right|}\left[\begin{array}{c}
-s \alpha s 2 \beta s \theta_{1} s \theta_{2}+c \alpha c \beta s\left(\theta_{2}-\theta_{1}\right) \\
-s \alpha c \beta s\left(\theta_{1}+\theta_{2}\right) \\
s 2 \alpha\left(c \theta_{1} c \theta_{2}+s^{2} \beta s \theta_{1} s \theta_{2}\right)+c 2 \alpha s \beta s\left(\theta_{1}-\theta_{2}\right)
\end{array}\right]
$$

Therefore, the following equalities can be written:

$$
\frac{-s \alpha s 2 \beta s \theta_{1} s \theta_{2}+c \alpha c \beta s\left(\theta_{2}-\theta_{1}\right)}{s \psi}=\frac{s \alpha c \beta s\left(\theta_{1}+\theta_{2}\right)}{s \phi}=\frac{s 2 \alpha\left(c\left(\theta_{2}-\theta_{1}\right)-c^{2} \beta s \theta_{1} s \theta_{2}\right)+c 2 \alpha s \beta s\left(\theta_{1}-\theta_{2}\right)}{\sqrt{1-s^{2} \phi-s^{2} \psi}}
$$

Let

$$
\begin{aligned}
& f_{1}\left(\theta_{1}, \theta_{2}, \phi, \psi\right)=\sqrt{1-s^{2} \phi-s^{2} \psi}\left[-s \alpha s 2 \beta s \theta_{1} s \theta_{2}+c \alpha c \beta s\left(\theta_{2}-\theta_{1}\right)\right] \\
& -s \psi\left\{s 2 \alpha\left[c\left(\theta_{2}-\theta_{1}\right)-c^{2} \beta s \theta_{1} s \theta_{2}\right]+c 2 \alpha s \beta s\left(\theta_{1}-\theta_{2}\right)\right\}=0 \\
& f_{2}\left(\theta_{1}, \theta_{2}, \phi, \psi\right)=\sqrt{1-s^{2} \phi-s^{2} \psi} s \alpha c \beta s\left(\theta_{1}+\theta_{2}\right) \\
& -s \phi\left\{s 2 \alpha\left[c\left(\theta_{2}-\theta_{1}\right)-c^{2} \beta s \theta_{1} s \theta_{2}\right]+c 2 \alpha s \beta s\left(\theta_{1}-\theta_{2}\right)\right\}=0
\end{aligned}
$$

Eqs. (21) is obtained by taking the time derivative of Eqs. (19) and (20).

$$
\begin{gathered}
\frac{d f_{1}}{d t}=\frac{\partial f_{1}}{\partial \theta_{1}} \dot{\theta}_{1}+\frac{\partial f_{1}}{\partial \theta_{2}} \dot{\theta}_{2}+\frac{\partial f_{1}}{\partial \phi} \dot{\phi}+\frac{\partial f_{1}}{\partial \psi} \dot{\psi}=0 \\
\frac{d f_{2}}{d t}=\frac{\partial f_{2}}{\partial \theta_{1}} \dot{\theta}_{1}+\frac{\partial f_{2}}{\partial \theta_{2}} \dot{\theta}_{2}+\frac{\partial f_{2}}{\partial \phi} \dot{\phi}+\frac{\partial f_{2}}{\partial \psi} \dot{\psi}=0
\end{gathered} \Rightarrow\left[\begin{array}{ll}
\frac{\partial f_{1}}{\partial \theta_{1}} & \frac{\partial f_{1}}{\partial \theta_{2}} \\
\frac{\partial f_{2}}{\partial \theta_{1}} & \frac{\partial f_{2}}{\partial \theta_{2}}
\end{array}\right]\left[\begin{array}{l}
\dot{\theta}_{1} \\
\dot{\theta}_{2}
\end{array}\right]=\left[\begin{array}{rr}
-\frac{\partial f_{1}}{\partial \phi} & -\frac{\partial f_{1}}{\partial \psi} \\
-\frac{\partial f_{2}}{\partial \phi} & -\frac{\partial f_{2}}{\partial \psi}
\end{array}\right]\left[\begin{array}{c}
\dot{\phi} \\
\dot{\psi}
\end{array}\right]
$$

where

$$
\begin{aligned}
& \frac{\partial f_{1}}{\partial \theta_{1}}=\sqrt{1-s^{2} \phi-s^{2} \psi}\left[-s \alpha s 2 \beta c \theta_{1} s \theta_{2}-c \alpha c \beta c\left(\theta_{2}-\theta_{1}\right)\right]-s \psi\left[s 2 \alpha\left(s\left(\theta_{2}-\theta_{1}\right)-c^{2} \beta c \theta_{1} s \theta_{2}\right)+c 2 \alpha s \beta c\left(\theta_{1}-\theta_{2}\right)\right] \\
& \frac{\partial f_{1}}{\partial \theta_{2}}=\sqrt{1-s^{2} \phi-s^{2} \psi}\left[-s \alpha s 2 \beta s \theta_{1} c \theta_{2}+c \alpha c \beta c\left(\theta_{2}-\theta_{1}\right)\right]-s \psi\left[s 2 \alpha\left(-s\left(\theta_{2}-\theta_{1}\right)-c^{2} \beta s \theta_{1} c \theta_{2}\right)-c 2 \alpha s \beta c\left(\theta_{1}-\theta_{2}\right)\right] \\
& \frac{\partial f_{1}}{\partial \phi}=\frac{-s \phi c \phi}{\sqrt{1-s^{2} \phi-s^{2} \psi}}\left[-s \alpha s 2 \beta s \theta_{1} s \theta_{2}+c \alpha c \beta s\left(\theta_{2}-\theta_{1}\right)\right] \\
& \frac{\partial f_{1}}{\partial \psi}=\frac{-s \psi c \psi}{\sqrt{1-s^{2} \phi-s^{2} \psi}}\left[-s \alpha s 2 \beta s \theta_{1} s \theta_{2}+c \alpha c \beta s\left(\theta_{2}-\theta_{1}\right)\right]-c \psi\left[s 2 \alpha\left(c\left(\theta_{2}-\theta_{1}\right)-c^{2} \beta s \theta_{1} s \theta_{2}\right)+c 2 \alpha s \beta s\left(\theta_{1}-\theta_{2}\right)\right] \\
& \frac{\partial f_{2}}{\partial \theta_{1}}=\sqrt{1-s^{2} \phi-s^{2} \psi} s \alpha c \beta c\left(\theta_{1}+\theta_{2}\right)-s \phi\left[\begin{array}{l}
s 2 \alpha\left(s\left(\theta_{2}-\theta_{1}\right)-c^{2} \beta c \theta_{1} s \theta_{2}\right) \\
+c 2 \alpha s \beta c\left(\theta_{1}-\theta_{2}\right)
\end{array}\right] \\
& \frac{\partial f_{2}}{\partial \theta_{2}}=\sqrt{1-s^{2} \phi-s^{2} \psi} s \alpha c \beta c\left(\theta_{1}+\theta_{2}\right)+s \phi\left[s 2 \alpha\left(s\left(\theta_{2}-\theta_{1}\right)+c^{2} \beta s \theta_{1} c \theta_{2}\right)+c 2 \alpha s \beta c\left(\theta_{1}-\theta_{2}\right)\right] \\
& \frac{\partial f_{2}}{\partial \phi}=-\frac{s \phi c \phi s \alpha c \beta s\left(\theta_{1}+\theta_{2}\right)}{\sqrt{1-s^{2} \phi-s^{2} \psi}}-c \phi\left[s 2 \alpha\left(c\left(\theta_{2}-\theta_{1}\right)-c^{2} \beta s \theta_{1} s \theta_{2}\right)+c 2 \alpha s \beta s\left(\theta_{1}-\theta_{2}\right)\right] \\
& \frac{\partial f_{2}}{\partial \psi}=-\frac{s \psi c \psi s \alpha c \beta s\left(\theta_{1}+\theta_{2}\right)}{\sqrt{1-s^{2} \phi-s^{2} \psi}}
\end{aligned}
$$

Some of the partial derivatives would become undefined if $\sqrt{1-s^{2} \phi-s^{2} \psi}=0$. This term being zero corresponds to the case where the endoscope axis is coplanar with the base plane. Such a configuration is quite far away from the selected workspace presented in Section 3.2, so it is guaranteed that such singularities are avoided. 
For $\beta=0$ (explained in Section 3.2.1), Eqs. (6) and (17)-(21) are simplified as follows:

$$
\begin{aligned}
& \left|\vec{n}_{1} \times \vec{n}_{2}\right|=\sqrt{s^{2} \theta_{2} c^{2} \theta_{1}+c^{2} \theta_{2} s^{2} \theta_{1}-2 c 2 \alpha c \theta_{1} s \theta_{1} c \theta_{2} s \theta_{2}+4 s^{2} \alpha c^{2} \alpha c^{2} \theta_{1} c^{2} \theta_{2}} \\
& \vec{w}=\left[\begin{array}{c}
s \psi \\
-s \phi \\
\sqrt{1-s^{2} \phi-s^{2} \psi}
\end{array}\right]=\frac{1}{\left|\vec{n}_{1} \times \vec{n}_{2}\right|}\left[\begin{array}{c}
c \alpha s\left(\theta_{2}-\theta_{1}\right) \\
-s \alpha s\left(\theta_{1}+\theta_{2}\right) \\
s 2 \alpha c \theta_{1} c \theta_{2}
\end{array}\right] \Rightarrow \frac{c \alpha s\left(\theta_{2}-\theta_{1}\right)}{s \psi}=\frac{s \alpha s\left(\theta_{1}+\theta_{2}\right)}{s \phi}=\frac{s 2 \alpha c \theta_{1} c \theta_{2}}{\sqrt{1-s^{2} \phi-s^{2} \psi}} \\
& \Rightarrow \begin{array}{l}
f_{1}\left(\theta_{1}, \theta_{2}, \phi, \psi\right)=\sqrt{1-s^{2} \phi-s^{2} \psi} s\left(\theta_{2}-\theta_{1}\right)-2 s \psi s \alpha c \theta_{1} c \theta_{2}=0 \\
f_{2}\left(\theta_{1}, \theta_{2}, \phi, \psi\right)=\sqrt{1-s^{2} \phi-s^{2} \psi} s\left(\theta_{1}+\theta_{2}\right)-2 s \phi c \alpha c \theta_{1} c \theta_{2}=0
\end{array} \\
& \frac{d f_{1}}{d t}=\frac{\partial f_{1}}{\partial \theta_{1}} \dot{\theta}_{1}+\frac{\partial f_{1}}{\partial \theta_{2}} \dot{\theta}_{2}+\frac{\partial f_{1}}{\partial \phi} \dot{\phi}+\frac{\partial f_{1}}{\partial \psi} \dot{\psi}=0 \\
& \frac{d f_{2}}{d t}=\frac{\partial f_{2}}{\partial \theta_{1}} \dot{\theta}_{1}+\frac{\partial f_{2}}{\partial \theta_{2}} \dot{\theta}_{2}+\frac{\partial f_{2}}{\partial \phi} \dot{\phi}+\frac{\partial f_{2}}{\partial \psi} \dot{\psi}=0 \quad\left[\begin{array}{cc}
\frac{\partial f_{1}}{\partial \theta_{1}} & \frac{\partial f_{1}}{\partial \theta_{2}} \\
\frac{\partial f_{2}}{\partial \theta_{1}} & \frac{\partial f_{2}}{\partial \theta_{2}}
\end{array}\right]\left[\begin{array}{l}
\dot{\theta}_{1} \\
\dot{\theta}_{2}
\end{array}\right]=\left[\begin{array}{cc}
-\frac{\partial f_{1}}{\partial \phi} & -\frac{\partial f_{1}}{\partial \psi} \\
-\frac{\partial f_{2}}{\partial \phi} & -\frac{\partial f_{2}}{\partial \psi}
\end{array}\right]\left[\begin{array}{c}
\dot{\phi} \\
\dot{\psi}
\end{array}\right]
\end{aligned}
$$

where

$$
\begin{aligned}
& \frac{\partial f_{1}}{\partial \theta_{1}}=-\sqrt{1-s^{2} \phi-s^{2} \psi} c\left(\theta_{2}-\theta_{1}\right)+2 s \psi s \alpha s \theta_{1} c \theta_{2} \quad \frac{\partial f_{1}}{\partial \theta_{2}}=\sqrt{1-s^{2} \phi-s^{2} \psi} c\left(\theta_{2}-\theta_{1}\right)+2 s \psi s \alpha c \theta_{1} s \theta_{2} \\
& \frac{\partial f_{1}}{\partial \phi}=\frac{-s \phi c \phi s\left(\theta_{2}-\theta_{1}\right)}{\sqrt{1-s^{2} \phi-s^{2} \psi}} \quad \frac{\partial f_{1}}{\partial \psi}=\frac{-s \psi c \psi s\left(\theta_{2}-\theta_{1}\right)}{\sqrt{1-s^{2} \phi-s^{2} \psi}}-2 c \psi s \alpha c \theta_{1} c \theta_{2} \\
& \frac{\partial f_{2}}{\partial \theta_{1}}=\sqrt{1-s^{2} \phi-s^{2} \psi} c\left(\theta_{1}+\theta_{2}\right)+2 s \phi c \alpha s \theta_{1} c \theta_{22} \quad \frac{\partial f_{2}}{\partial \theta_{2}}=\sqrt{1-s^{2} \phi-s^{2} \psi} c\left(\theta_{1}+\theta_{2}\right)+2 s \phi c \alpha c \theta_{1} s \theta_{2} \\
& \frac{\partial f_{2}}{\partial \phi}=-\frac{s \phi c \phi s\left(\theta_{1}+\theta_{2}\right)}{\sqrt{1-s^{2} \phi-s^{2} \psi}}-2 c \phi c \alpha c \theta_{1} c \theta_{22} \quad \frac{\partial f_{2}}{\partial \psi}=-\frac{s \psi c \psi s\left(\theta_{1}+\theta_{2}\right)}{\sqrt{1-s^{2} \phi-s^{2} \psi}}
\end{aligned}
$$

Rearranging Eq. (15):

$$
f_{3}\left(d, \psi, \phi, \theta_{3}\right)=d^{2}-2 d\left[f c \gamma+a_{3} c\left(\theta_{3}-\gamma\right)\right]+\left[f c \gamma+a_{3} c\left(\theta_{3}-\gamma\right)\right]^{2}+\left[c_{3}-f s \gamma+a_{3} s\left(\theta_{3}-\gamma\right)\right]^{2}-b_{3}{ }^{2}=0
$$

where $\gamma=\frac{\pi}{2}-\psi$ as can be found from Eq. (7) for $\beta=0$. Therefore, $\mathrm{f}_{3}$ does not depend on $\phi$. Similarly, Eqs. (28) and (29) are found using the total derivative in Eq. (27):

$$
\begin{aligned}
& \frac{\partial f_{3}}{\partial d} \dot{d}+\frac{\partial f_{3}}{\partial \psi} \dot{\psi}+\frac{\partial f_{3}}{\partial \theta_{3}} \dot{\theta}_{3}=0 \\
& \dot{\theta}_{3}=-\left(\frac{\partial f_{3}}{\partial d} \dot{d}+\frac{\partial f_{3}}{\partial \psi} \dot{\psi}\right) / \frac{\partial f_{3}}{\partial \theta_{3}}
\end{aligned}
$$

or

$$
\dot{d}=-\left(\frac{\partial f_{3}}{\partial \psi} \dot{\psi}+\frac{\partial f_{3}}{\partial \theta_{3}} \dot{\theta}_{3}\right) / \frac{\partial f_{3}}{\partial d}
$$

where

$$
\begin{aligned}
& \frac{\partial f_{3}}{\partial d}=2\left[d-f c \gamma-a_{3} c\left(\theta_{3}-\gamma\right)\right] \frac{\partial f_{3}}{\partial \psi}=-\frac{\partial f_{3}}{\partial \gamma}=-2 d\left[f s \gamma-a_{3} s\left(\theta_{3}-\gamma\right)\right]+2 c_{3}\left[f c \gamma+a_{3} c\left(\theta_{3}-\gamma\right)\right] \\
& \frac{\partial f_{3}}{\partial \theta_{3}}=2 a_{3}\left[s\left(\theta_{3}-\gamma\right)(d-f c \gamma)+c\left(\theta_{3}-\gamma\right)\left(c_{3}-f s \gamma\right)\right]
\end{aligned}
$$

The angular speed in Eq. (28) would be undefined if $\partial \mathrm{f}_{3} / \partial \theta_{3}=0$, which happens when $A_{3}, B_{3}$ and $C_{3}$ in Fig. 4 become collinear. As explained in Section 3.1.2, the manipulator never attains this singular configuration within the desired workspace. The speed in Eq. (29) would be undefined if $\partial \mathrm{f}_{3} / \partial d=0$, which happens if $B_{3}, C_{3}$ and $E$ in Fig. 4 become collinear. As explained in Section 3.1.1, the manipulator is designed to never attain this configuration. 
The relationship between joint space and task space velocities are constructed as:

$$
\left[\begin{array}{ccc}
\frac{\partial f_{1}}{\partial \theta_{1}} & \frac{\partial f_{1}}{\partial \theta_{2}} & 0 \\
\frac{\partial f_{2}}{\partial \theta_{1}} & \frac{\partial f_{2}}{\partial \theta_{2}} & 0 \\
0 & 0 & \frac{\partial f_{3}}{\partial \theta_{3}}
\end{array}\right]\left[\begin{array}{c}
\dot{\theta}_{1} \\
\dot{\theta}_{2} \\
\dot{\theta}_{3}
\end{array}\right]=\left[\begin{array}{ccc}
-\frac{\partial f_{1}}{\partial \phi} & -\frac{\partial f_{1}}{\partial \psi} & 0 \\
-\frac{\partial f_{2}}{\partial \phi} & -\frac{\partial f_{2}}{\partial \psi} & 0 \\
0 & -\frac{\partial f_{3}}{\partial \psi} & -\frac{\partial f_{3}}{\partial d}
\end{array}\right]\left[\begin{array}{c}
\dot{\phi} \\
\dot{\psi} \\
\dot{d}
\end{array}\right]
$$

For the part involving the orientation of the end-effector axis only,

$$
\left[\begin{array}{ll}
\frac{\partial f_{1}}{\partial \theta_{1}} & \frac{\partial f_{1}}{\partial \theta_{2}} \\
\frac{\partial f_{2}}{\partial \theta_{1}} & \frac{\partial f_{2}}{\partial \theta_{2}}
\end{array}\right]\left[\begin{array}{l}
\dot{\theta}_{1} \\
\dot{\theta}_{2}
\end{array}\right]=\left[\begin{array}{cc}
-\frac{\partial f_{1}}{\partial \phi} & -\frac{\partial f_{1}}{\partial \psi} \\
-\frac{\partial f_{2}}{\partial \phi} & -\frac{\partial f_{2}}{\partial \psi}
\end{array}\right]\left[\begin{array}{c}
\dot{\phi} \\
\dot{\psi}
\end{array}\right] \Rightarrow\left[\begin{array}{c}
\dot{\theta}_{1} \\
\dot{\theta}_{2}
\end{array}\right]=\hat{J}\left[\begin{array}{c}
\dot{\phi} \\
\dot{\psi}
\end{array}\right]
$$

where

$$
\hat{J}=\frac{1}{\frac{\partial f_{1}}{\partial \theta_{1}} \frac{\partial f_{2}}{\partial \theta_{2}}-\frac{\partial f_{2}}{\partial \theta_{1}} \frac{\partial f_{1}}{\partial \theta_{2}}}\left[\begin{array}{cc}
\frac{\partial f_{2}}{\partial \theta_{2}} & -\frac{\partial f_{1}}{\partial \theta_{2}} \\
-\frac{\partial f_{2}}{\partial \theta_{1}} & \frac{\partial f_{1}}{\partial \theta_{1}}
\end{array}\right]\left[\begin{array}{cc}
-\frac{\partial f_{1}}{\partial \phi} & -\frac{\partial f_{1}}{\partial \psi} \\
-\frac{\partial f_{2}}{\partial \phi} & -\frac{\partial f_{2}}{\partial \psi}
\end{array}\right]=\frac{1}{\Delta}\left[\begin{array}{ll}
J_{11} & J_{12} \\
J_{21} & J_{22}
\end{array}\right]
$$

with $J_{11}=\frac{\partial f_{1}}{\partial \theta_{2}} \frac{\partial f_{2}}{\partial \phi}-\frac{\partial f_{2}}{\partial \theta_{2}} \frac{\partial f_{1}}{\partial \phi}, \mathrm{J}_{12}=\frac{\partial f_{1}}{\partial \theta_{2}} \frac{\partial f_{2}}{\partial \psi}-\frac{\partial f_{2}}{\partial \theta_{2}} \frac{\partial f_{1}}{\partial \psi}, \mathrm{J}_{21}=\frac{\partial f_{2}}{\partial \theta_{1}} \frac{\partial f_{1}}{\partial \phi}-\frac{\partial f_{1}}{\partial \theta_{1}} \frac{\partial f_{2}}{\partial \phi}, \mathrm{J}_{22}=\frac{\partial f_{2}}{\partial \theta_{1}} \frac{\partial f_{1}}{\partial \psi}-\frac{\partial f_{1}}{\partial \theta_{1}} \frac{\partial f_{2}}{\partial \psi}$ and $\Delta=\frac{\partial f_{1}}{\partial \theta_{1}} \frac{\partial f_{2}}{\partial \theta_{2}}-\frac{\partial f_{2}}{\partial \theta_{1}} \frac{\partial f_{1}}{\partial \theta_{2}}$. As it is explained in Section 3.2, the target workspace is far from singularities, so we do not get into details of singularity analysis or determine the boundaries of the reachable workspace in this study. Here we just specify that for $\alpha=\pi / 4$ (the chosen value in Section 3.2.1), singularity occurs for $\theta_{1}= \pm \pi / 2$ and $\theta_{2}= \pm \pi / 2$ in the joint space. These configurations correspond to the case where the side leg planes (and hence the middle leg plane as well) are coplanar with the base plane. Obviously, for our application, the manipulator never goes to this singular pose. Also, there are possible singularities due to the passive joints in the side legs (extended and folded configurations of links $A_{i} B_{i}$ and $B_{i} C_{i}$ for $i=1,2-$ see Fig. $2 b$ ), but for the selected target workspace, the link lengths are selected to avoid such singularities. For evaluating the condition number for $\hat{\jmath}$,

$$
\begin{aligned}
& \hat{J}^{T} \hat{J}=\frac{1}{\Delta^{2}}\left[\begin{array}{cc}
J_{11}{ }^{2}+\mathrm{J}_{21}{ }^{2} & J_{11} \mathrm{~J}_{12}+\mathrm{J}_{21} \mathrm{~J}_{22} \\
J_{11} \mathrm{~J}_{12}+\mathrm{J}_{21} \mathrm{~J}_{22} & \mathrm{~J}_{12}{ }^{2}+\mathrm{J}_{22}{ }^{2}
\end{array}\right] \\
& |\hat{J} \hat{J}-\lambda \hat{I}|=\frac{1}{\Delta^{2}}\left|\begin{array}{cc}
J_{11}{ }^{2}+\mathrm{J}_{21}{ }^{2}-\Delta^{2} \lambda & J_{11} \mathrm{~J}_{12}+\mathrm{J}_{21} \mathrm{~J}_{22} \\
J_{11} \mathrm{~J}_{12}+\mathrm{J}_{21} \mathrm{~J}_{22} & \mathrm{~J}_{12}{ }^{2}+\mathrm{J}_{22}{ }^{2}-\Delta^{2} \lambda
\end{array}\right| \\
& =\left[\left(J_{11}{ }^{2}+\mathrm{J}_{21}{ }^{2}-\Delta^{2} \lambda\right)\left(\mathrm{J}_{12}{ }^{2}+\mathrm{J}_{22}{ }^{2}-\Delta^{2} \lambda\right)-\left(J_{11} \mathrm{~J}_{12}+\mathrm{J}_{21} \mathrm{~J}_{22}\right)^{2}\right] / \Delta^{2} \\
& =\Delta^{2} \lambda^{2}-\left({J_{11}}^{2}+\mathrm{J}_{12}{ }^{2}+\mathrm{J}_{21}{ }^{2}+\mathrm{J}_{22}{ }^{2}\right) \lambda+1=0
\end{aligned}
$$

The eigenvalues of $\hat{J}^{T} \hat{J}$ are

$$
\lambda_{1,2}=\frac{J_{11}{ }^{2}+\mathrm{J}_{12}{ }^{2}+\mathrm{J}_{21}{ }^{2}+\mathrm{J}_{22}{ }^{2} \mp \sqrt{\left(J_{11}{ }^{2}+\mathrm{J}_{12}{ }^{2}+\mathrm{J}_{21}{ }^{2}+\mathrm{J}_{22}{ }^{2}\right)^{2}-4 \Delta^{2}}}{2 \Delta^{2}}
$$

Then, the condition number of $\hat{J}$ is found as:

$$
\kappa=\sqrt{\frac{\max \left(\left|\lambda_{1}\right|,\left|\lambda_{2}\right|\right)}{\min \left(\left|\lambda_{1}\right|,\left|\lambda_{2}\right|\right)}}
$$

The condition number in Eq. (36) is to be used in the kinematic design of the manipulator considering optimal force transmission characteristics regarding the $\theta_{1}$ and $\theta_{2}$ inputs, and orientation of the end-effector. For the input angle $\theta_{3}$ and the translation $\mathrm{d}$ of the end-effector, the transmission angle of a slider-crank mechanism in the middle leg plane is used for design.

\subsection{Kinematic design}

For the kinematic design, firstly the workspace dimensions of the manipulator need to be defined. Since this manipulator is to be used for transnasal pituitary gland surgeries, the workspace is the nasal cavity of a patient. To identify the motion limits inside the nasal cavity, first, the computed tomography scans of a group of patients were investigated by Dede et al. 


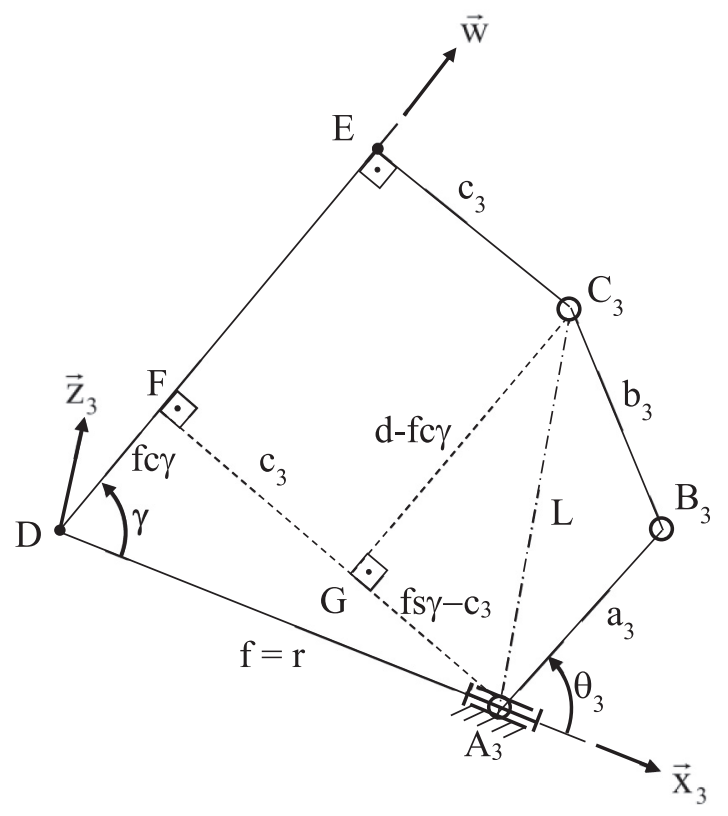

Fig. 5. Dimensions of the slider-crank mechanism in the middle leg.

[5]. The nominal dimensions of the cavity are measured and then, a specifically designed measurement tool with an inertial measurement unit was mounted on an endoscope. This endoscope is used on a cadaver with nominal nasal cavity dimensions and it is moved around the borders of the nasal cavity. The measured rotational motions are recorded and later, they were extended to cover the $99.7 \%$ confidence interval. The results indicate that the endoscope is moved $33^{\circ}$ for pitch and $23^{\circ}$ for yaw motion about the RCM and $95 \mathrm{~mm}$ for heave motion through the RCM for a transnasal operation. This means the mechanism should have at least $\Delta \psi=33^{\circ}, \Delta \phi=23^{\circ}$ and $\Delta d=95 \mathrm{~mm}$ of motion ranges. Consequently, the workspace ranges of the manipulator are chosen as $\Delta \psi=40^{\circ}, \Delta \phi=30^{\circ}$ and $\Delta d=100 \mathrm{~mm}$.

In Section 3.2.1, the transmission characteristics of pitch and yaw motions are optimized based on the condition number and angles $\alpha$ and $\beta$ are selected. The link-length dimensions of the side legs do not have a significant effect on the input/output relationship of the manipulator because, except the $\mathrm{R}$ joints on the base platform, all the joints on the side legs are passive. However, a dimensional design is performed for the middle leg in Section 3.2.2 and the same link length dimensions are used for the side legs.

\subsubsection{Condition number optimization for pitch and yaw motions}

By applying Eq. (36), the condition numbers for $5^{\circ}<\alpha<85^{\circ}$ and $-45^{\circ}<\beta<45^{\circ}$ are computed and tabulated in Table 1. Each cell in the table shows the maximum condition number is found in the workspace ranges: $-15^{\circ}<\phi<15^{\circ}$ and $-20^{\circ}<\psi<20^{\circ}$ for the specific $\alpha$ and $\beta$ angles. The cases where $\alpha=0^{\circ}$ and $\alpha=90^{\circ}$ correspond to the cases where all or some of the fixed $\mathrm{R}$ joint axes on the base platform coincide, so these values are disregarded and $\alpha=0^{\circ}$ and $\alpha=90^{\circ}$ columns are not presented in the table. As highlighted in the table, the minimum condition number is obtained as 1.064 for $\alpha=45^{\circ}$ and $\beta=0^{\circ}$.

Once the structural parameters are selected as $\alpha=45^{\circ}$ and $\beta=0^{\circ}$, the condition numbers for the workspace of the manipulator are tabulated with the shaded gradation in Table 2. As can be seen, the maximum condition number value 1.064 is obtained for $\phi=0^{\circ}$ and $\psi= \pm 20^{\circ}$, which is still very close to 1 . This means that the mechanism shows almost fully-isotropic characteristics for pitch and yaw motions. So, the structural parameters $\alpha$ and $\beta$ are optimized in terms of transmission characteristics.

\subsubsection{Slider-crank design for the middle leg}

In the middle plane in Fig. 3, the middle leg can be considered as a slider-crank mechanism with a crank $A_{3} B_{3}$, a connecting rod $B_{3} C_{3}$ and link $C_{3}$ ED sliding along DE direction (Fig. 5). The plane of this slider-crank mechanism can rotate about the $\mathrm{x}_{3}$-axis. Also, the angle of the sliding direction of the slider can be changed by angle $\gamma$. For this slider-crank mechanism, constant $\mathrm{DEC}_{3}$ angle is chosen as $90^{\circ}$ for maximum force transmission. Since $\beta$ is previously selected as $0, h=0$ and $f=r$ (see Fig. 2). The choice of $c_{3}$ is arbitrary. However, numerical simulations show that $c_{3}=r$ results in better transmission characteristics. Choice of $r$ depends on the application. For our application, it is determined based on the average size of a patient's head. Consequently, only parameters $a_{3}$ and $b_{3}$ are to be designed. 


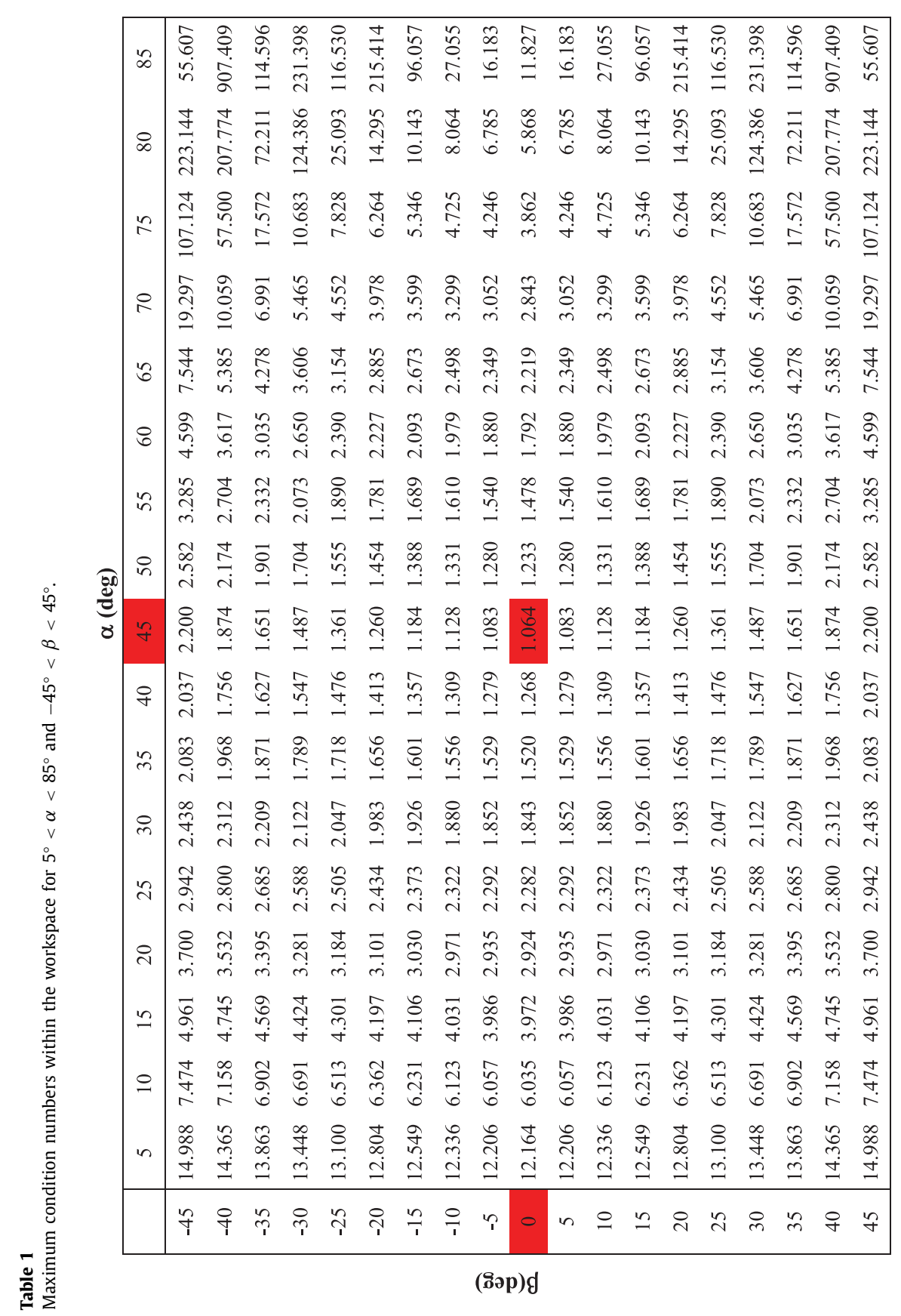


Table 2

Condition numbers within the workspace for $\alpha=45^{\circ}$ and $\beta=0^{\circ}$.

\begin{tabular}{|c|c|c|c|c|c|c|c|c|c|c|c|}
\hline \multicolumn{12}{|c|}{$\phi(\operatorname{deg})$} \\
\hline & -15 & -12 & -9 & -6 & -3 & 0 & 3 & 6 & 9 & 12 & 15 \\
\hline-20.00 & 1.028 & 1.041 & 1.051 & 1.059 & 1.063 & 1.064 & 1.063 & 1.059 & 1.051 & 1.041 & 1.028 \\
\hline-15.56 & 1.003 & 1.015 & 1.025 & 1.032 & 1.037 & 1.038 & 1.037 & 1.032 & 1.025 & 1.015 & 1.003 \\
\hline-11.11 & 1.016 & 1.003 & 1.007 & 1.014 & 1.018 & 1.019 & 1.018 & 1.014 & 1.007 & 1.003 & 1.016 \\
\hline-6.67 & 1.028 & 1.015 & 1.006 & 1.001 & 1.005 & 1.007 & 1.005 & 1.001 & 1.006 & 1.015 & 1.028 \\
\hline-2.22 & 1.035 & 1.022 & 1.012 & 1.005 & 1.001 & 1.001 & 1.001 & 1.005 & 1.012 & 1.022 & 1.035 \\
\hline 2.22 & 1.035 & 1.022 & 1.012 & 1.005 & 1.001 & 1.001 & 1.001 & 1.005 & 1.012 & 1.022 & 1.035 \\
\hline 6.67 & 1.028 & 1.015 & 1.006 & 1.001 & 1.005 & 1.007 & 1.005 & 1.001 & 1.006 & 1.015 & 1.028 \\
\hline 11.11 & 1.016 & 1.003 & 1.007 & 1.014 & 1.018 & 1.019 & 1.018 & 1.014 & 1.007 & 1.003 & 1.016 \\
\hline 15.56 & 1.003 & 1.015 & 1.025 & 1.032 & 1.037 & 1.038 & 1.037 & 1.032 & 1.025 & 1.015 & 1.003 \\
\hline 20.00 & 1.028 & 1.041 & 1.051 & 1.059 & 1.063 & 1.064 & 1.063 & 1.059 & 1.051 & 1.041 & 1.028 \\
\hline
\end{tabular}

\begin{tabular}{c|c}
$\mathbf{a}_{3}$ & 135 \\
\hline $\mathbf{b}_{3}$ & 195 \\
\hline $\mathbf{c}_{\mathbf{3}}$ & 200 \\
\hline $\mathbf{r}$ & 200 \\
\hline $\mathbf{h}$ & 0 \\
\hline $\mathbf{f}$ & 200 \\
\hline $\boldsymbol{\beta}$ & 0 \\
\hline$\Delta \boldsymbol{\gamma}$ & 40 \\
\hline $\mathbf{d}_{\mathbf{0}}$ & 250 \\
\hline$\Delta \mathbf{d}$ & 100 \\
\hline$\Delta \mathbf{d}_{\mathbf{e}}$ & 20 \\
\hline $\mathbf{b + a} \geq$ & 318,6 \\
\hline $\mathbf{b}-\mathbf{a} \leq$ & 82,5 \\
\hline $\mathbf{a}_{\min }$ & 118,1 \\
\hline $\mathbf{b} \geq$ & 158,6 \\
\hline $\mathbf{b} \leq$ & 242,5
\end{tabular}

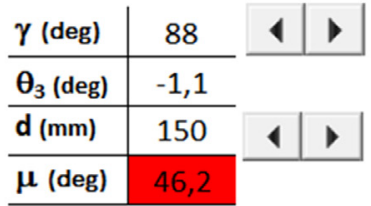

\begin{tabular}{ccc} 
& $\mathbf{x}$ & $\mathbf{y}$ \\
\cline { 2 - 3 } $\mathbf{D}$ & 0 & 0 \\
\hline $\mathbf{A}_{\mathbf{3}}$ & 200 & 0 \\
\hline $\mathbf{B}_{3}$ & 335,0 & $-2,5$ \\
\hline $\mathbf{C}_{\mathbf{3}}$ & 205,1 & 142,9 \\
\hline $\mathbf{E}$ & 5,2 & 149,9 \\
\hline $\mathbf{T}$ & $-3,5$ & $-99,9$ \\
\hline
\end{tabular}

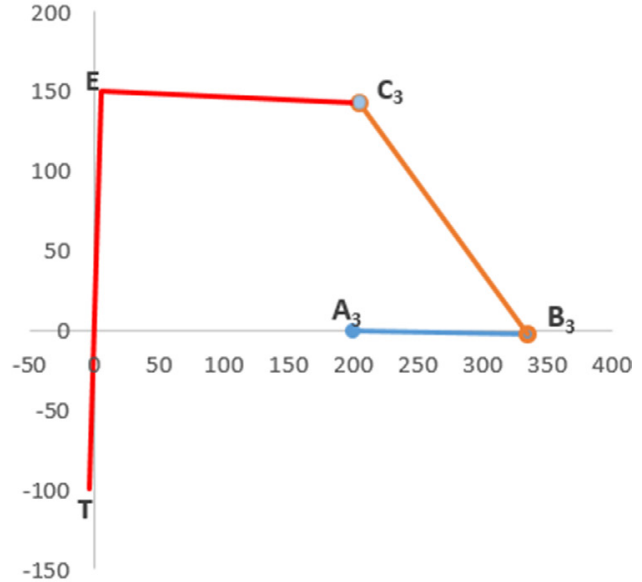

Fig. 6. Design sheet of the slider-crank mechanism in Microsoft Excel.

For the initial slider displacement, the distance between the center of mass and the tip of the endoscope is taken as a reference value. For the specific endoscope that is used in this surgery, this distance is $d_{0}=250 \mathrm{~mm}$. As depicted in Fig. 5 , for the slider-crank mechanism,

(1) Eccentricity $\left|A_{3} G\right|: f s \gamma-c_{3}$

(2) Initial slider displacement: $\mathrm{d}_{0}=250 \mathrm{~mm}$

(3) Stroke: $\Delta d=100 \mathrm{~mm}$

(4) Effective workspace limits (at the end of the stroke): $\pm \Delta \mathrm{d}_{\mathrm{e}}=20 \mathrm{~mm}$

(5) Zero position at the center of the workspace: $d=\mathrm{d}_{0}-\Delta d+\Delta \mathrm{d}_{\mathrm{e}}=170 \mathrm{~mm}$

To satisfy the workspace limits, $\Delta d=100 \mathrm{~mm}$ and $\Delta \gamma=40^{\circ}: \gamma_{\min }=70^{\circ} \leq \gamma \leq 110^{\circ}=\gamma_{\max }$. The following limiting conditions on the link lengths in the limit configurations need to be taken into consideration:

$$
\begin{gathered}
b_{3}+a_{3} \geq \sqrt{\left(f s \gamma_{\max }-c_{3}\right)^{2}+\left(d_{0}-f c \gamma_{\max }\right)^{2}}=L_{\max } \\
b_{3}-a_{3} \leq \sqrt{\left(f s \gamma_{\min }-c_{3}\right)^{2}+\left(d_{0}-\Delta d-f c \gamma_{\min }\right)^{2}}=L_{\min }
\end{gathered}
$$




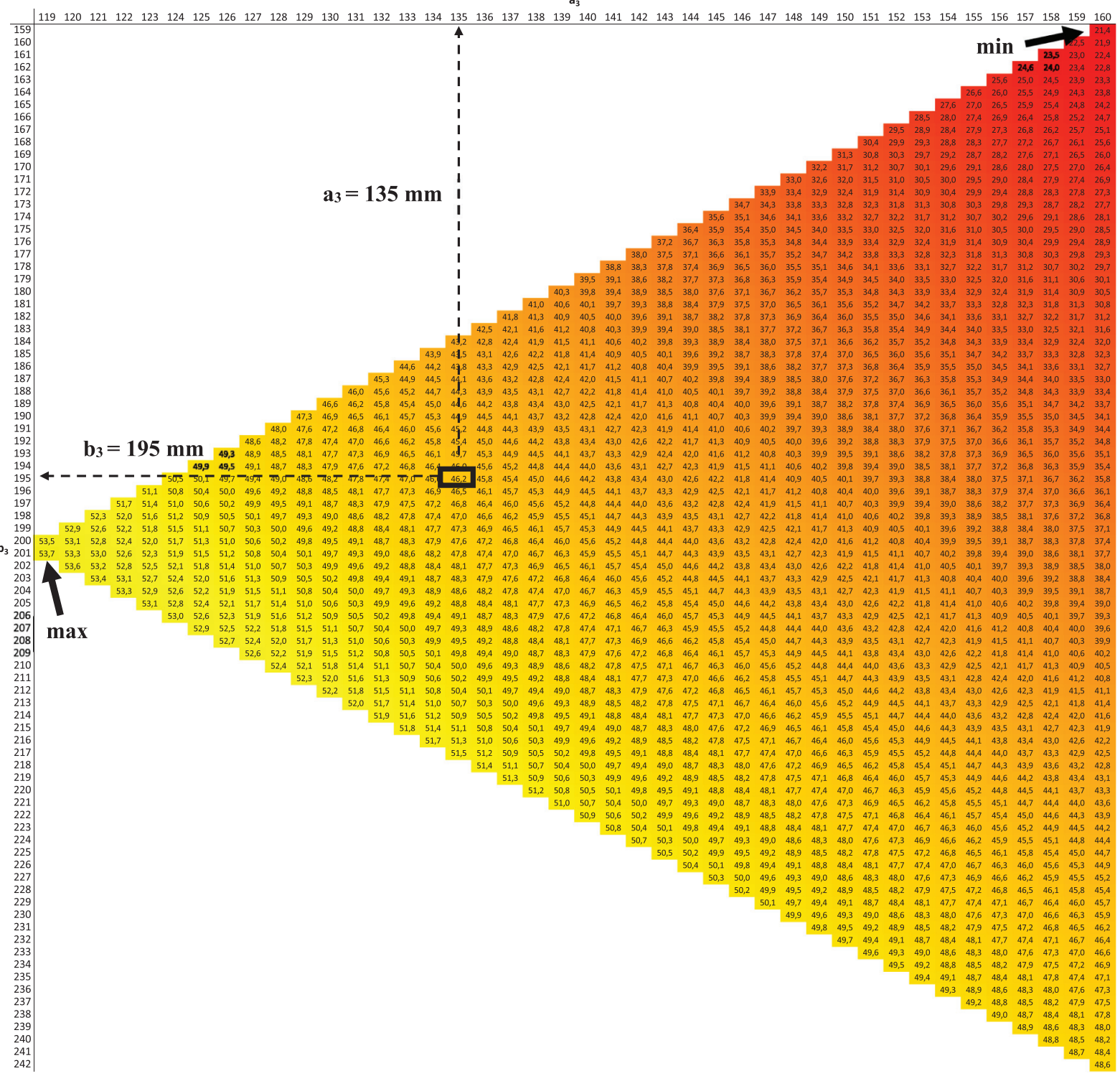

Fig. 7. Minimum transmission angles for slider-crank mechanism within its workspace for $119 \mathrm{~mm} \leq \mathrm{a}_{3} \leq 160 \mathrm{~mm}$ and $159 \mathrm{~mm} \leq \mathrm{b}_{3} \leq 242 \mathrm{~mm}$.

From Eqs. (37),

$$
a_{3} \geq \frac{L_{\max }-L_{\min }}{2} \text { and } a_{3}+L_{\min } \geq b_{3} \geq L_{\max }-a_{3}
$$

The formulations are implemented in Microsoft Excel and the slider-crank mechanism is designed. A snapshot of the Excel design sheet is depicted in Fig. 6. On the left side of Fig. 6, the design parameters and conditions are listed. After specifying $a_{3}$ and $b_{3}$ values, $\gamma$ angle and $d$ values can be changed with corresponding spin buttons to compute the transmission angle $\mu$ of the slider-crank mechanism. Due to footprint limitations, $\mathrm{a}_{3}$ length should be less than $160 \mathrm{~mm}$. From Eqs. (38) $118.1^{\circ} \leq \mathrm{a}_{3} \leq 160^{\circ}$ and $158.6^{\circ} \leq \mathrm{b}_{3} \leq 242.5^{\circ}$. Thus, for these dimension ranges, the minimum transmission angles are found throughout the workspace of the slider-crank mechanism and the results are tabulated with shaded gradation in Fig. 7. In Fig. 7, the horizontal axis represents $a_{3}$ values from $119 \mathrm{~mm}$ to $160 \mathrm{~mm}$ and the vertical axis represents $b_{3}$ values from $159 \mathrm{~mm}$ to $242 \mathrm{~mm}$. The maximum value, $53.7^{\circ}$ is obtained towards the middle left side of the graph while the minimum value of $21.4^{\circ}$ is found on the top right-hand corner. The results for infeasible dimensions are not presented. After considering the constructional constraints such as interference of the links $a_{3}$ and $b_{3}$ with each other and collision of the link $a_{3}$ with the motor that drives the slider-crank mechanism, the optimal solution with highest minimum transmission 


\begin{tabular}{|c|c|c|c|c|}
\hline \multicolumn{2}{|c|}{ Design Parameters } & \multirow[b]{2}{*}{4} & \multirow{2}{*}{\multicolumn{2}{|c|}{ v }} \\
\hline$\alpha$ (deg) & 45 & & & \\
\hline$\beta$ (deg) & 0 & 1 & - & \\
\hline$r(m m)$ & 200 & 4 & - & \\
\hline$h(\mathrm{~mm})$ & 0 & & & \\
\hline$f(m m)$ & 200 & & & \\
\hline$a_{3}(\mathrm{~mm})$ & 135 & 4 & D & 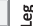 \\
\hline$b_{3}(\mathrm{~mm})$ & 195 & 1 & 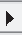 & $\frac{0}{2}$ \\
\hline$c_{3}(\mathrm{~mm})$ & 200 & 4 & D & in \\
\hline$a_{1,2}(\mathrm{~mm})$ & 135 & & & \\
\hline $\mathrm{b}_{1,2}(\mathrm{~mm})$ & 195 & & & \\
\hline$c_{1,2}(\mathrm{~mm})$ & 200 & iँ & & \\
\hline
\end{tabular}

\begin{tabular}{|c|c|}
\hline$\delta f_{1} / \delta \theta_{1}$ & 0,3845 \\
\hline$\delta f_{1} / \delta \theta_{2}$ & $-0,2042$ \\
\hline$\delta f_{2} / \delta \theta_{1}$ & 0,5831 \\
\hline$\delta f_{2} / \delta \theta_{2}$ & 0,6792 \\
\hline$\delta f_{1} / \delta \phi$ & $-0,1438$ \\
\hline$\delta f_{1} / \delta \psi$ & 0,4201 \\
\hline$\delta f_{2} / \delta \phi$ & $-0,9862$ \\
\hline$\delta f_{2} / \delta \psi$ & $-0,0754$ \\
\hline
\end{tabular}

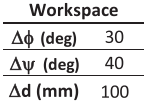

$\Delta \mathrm{d}(\mathrm{mm}) \quad 100$

\begin{tabular}{|c|c|c|c|c|c|c|c|c|c|c|c|}
\hline \multicolumn{6}{|c|}{ Forward Kinematics } & \multicolumn{6}{|c|}{ Inverse Kinematics } \\
\hline \multicolumn{3}{|c|}{ Actuation Inputs } & \multicolumn{3}{|c|}{ Orientation Outputs } & \multicolumn{3}{|c|}{ Orientation Inputs } & \multicolumn{3}{|c|}{ Actuation Outputs } \\
\hline & deg & $\mathrm{rad}$ & & $\mathrm{rad}$ & deg & & deg & $\mathrm{rad}$ & & rad & deg \\
\hline$\theta_{1}$ & 12 & 0,2094 & $\psi$ & 0,1604 & 9,1884 & $\psi$ & 9,1884 & 0,1604 & $\theta_{1}$ & 0,2094 & 12 \\
\hline$\theta_{2}$ & 25 & 0,4363 & $\phi$ & 0,4414 & 25,2900 & $\phi$ & 25,2900 & 0,4414 & $\theta_{2}$ & 0,4363 & 25 \\
\hline$\theta_{3}$ & 36 & 0,6283 & $d(m m)$ & 299,3315 & & $\mathrm{~d}(\mathrm{~mm})$ & 299,3315 & & $\theta_{3}$ & 0,6283 & 36 \\
\hline
\end{tabular}

$\begin{array}{cc}0,3845 & -0,2042 \\ 0,5831 & 0,6792 \\ 0 & 0\end{array}-5039$

\begin{tabular}{c|}
0 \\
0 \\
503910
\end{tabular}

\begin{tabular}{l}
$\delta \theta_{1}$ \\
$\delta \theta_{2}$ \\
$\delta \theta_{3}$ \\
\hline
\end{tabular}

\begin{tabular}{|ccc|}
\hline 0,1438 & $-0,4201$ & 0 \\
0,9862 & 0,0754 & 0 \\
0 & $-124072,3$ & $-343,244$ \\
\hline
\end{tabular}

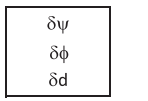

\begin{tabular}{|ccc|}
\hline 0,6443 & 0,6349 & $-3,4 \mathrm{E}-18$ \\
$-0,6947$ & 0,7033 & $5 \mathrm{E}-17$ \\
251,1228 & $-254,2387$ & 146,8079 \\
\hline
\end{tabular}

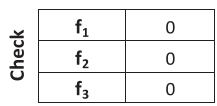
Jacobian Matrix

\begin{tabular}{|c|c|}
\hline$\delta f_{3} / \delta \theta_{3}$ & -50391 \\
\hline$\delta f_{3} / \delta d$ & 343 \\
\hline$\delta f_{3} / \delta \gamma$ & -124072 \\
\hline$\delta \gamma / \delta \phi$ & 0 \\
\hline$\delta \gamma / \delta \psi$ & 1 \\
\hline$\delta f_{3} / \delta \phi$ & 0 \\
\hline$\delta f_{3} / \delta \psi$ & -124072 \\
\hline
\end{tabular}

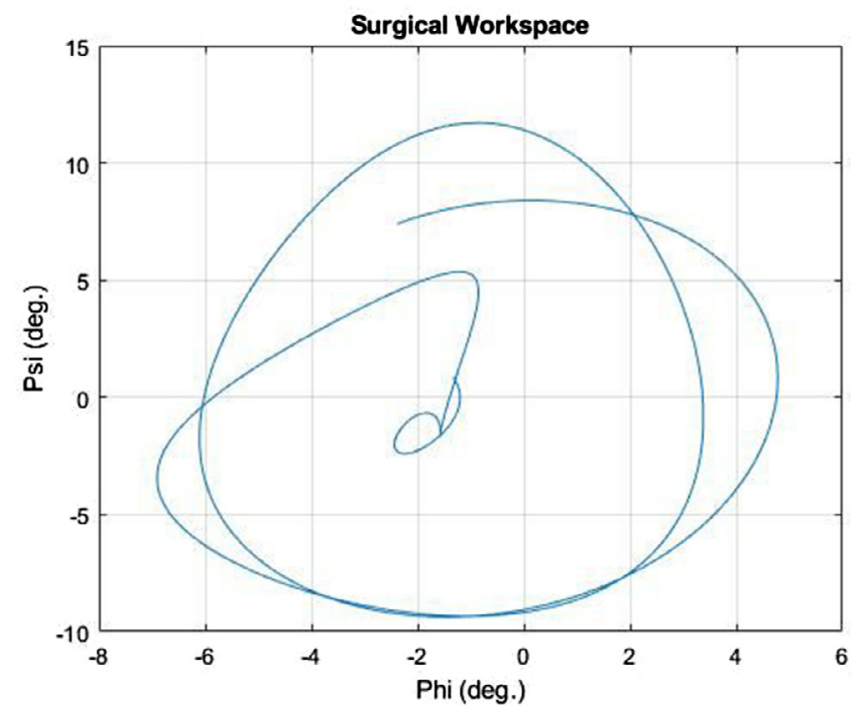

Fig. 9. The rotational workspace of the endoscope inside the surgical area of a cadaver.

angle is chosen for $\mathrm{a}_{3}=135 \mathrm{~mm}$ and $\mathrm{b}_{3}=195 \mathrm{~mm}$. For these dimensions, the worst transmission angle value is obtained as $\mu_{\min } \cong 46.2^{\circ}$ when $\gamma=88^{\circ}$ and $d=150 \mathrm{~mm}$. This configuration is presented in Fig. 6 .

All kinematic formulations are crosschecked by a study in Microsoft Excel before starting detailed constructional design. In Fig. 8, design parameters are provided on the top left side. Then, direct and inverse kinematics analyses are performed. As can be seen, the same arbitrary input values in the direct kinematics can be re-obtained as a result of the inverse kinematics (blue highlights). By using the derivatives presented on the bottom left side of the figure, the Jacobian matrix is obtained and highlighted with light green color. The Jacobian matrix shows that pitch $(\psi)$ and yaw $(\phi)$ motions of the end-effector are decoupled from the heave (d) motion due to the zero elements in the first and second rows of the third column. However, the heave motion is coupled with other motions due to the non-zero off-diagonal elements in the last row.

\section{Kinematic simulations}

A simulation test is carried out to verify the inverse kinematics and gain insight into the dynamics of the RCM mechanism. The simulation model is developed by following the procedure defined in [4]. The CAD models of the parts (i.e. links, joint structures) of the mechanism (presented in Section 5) are first assembled in SolidWorks with the suitable mating procedures to create the correct joint structures. Then, the assembled model is translated from SolidWorks to Matlab via the 

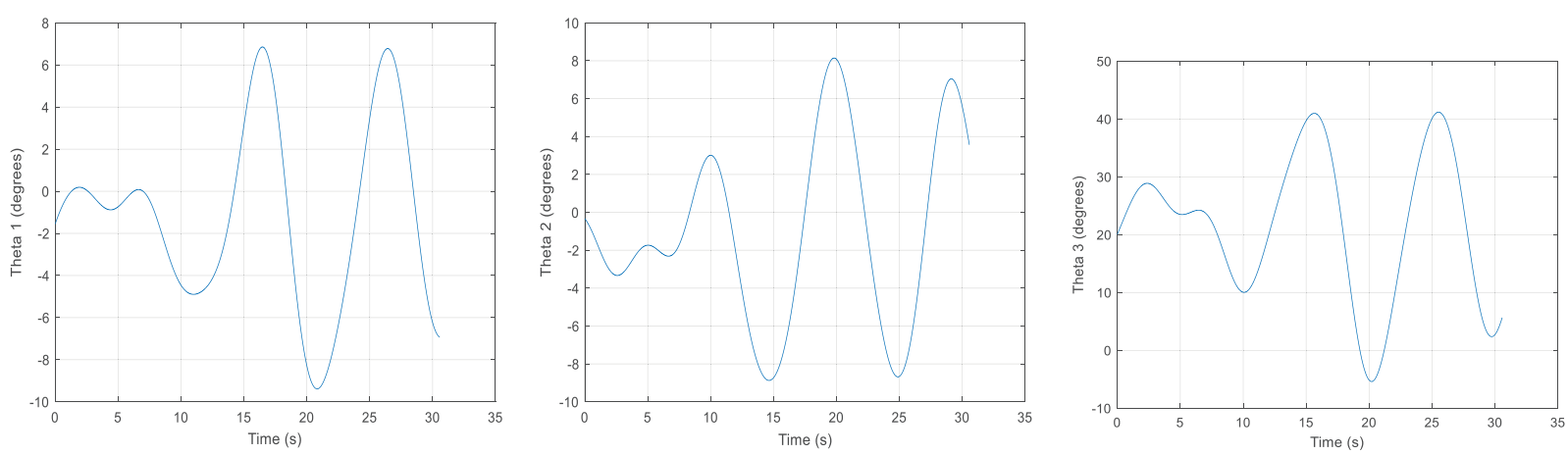

Fig. 10. Joint trajectories during the simulation tests.

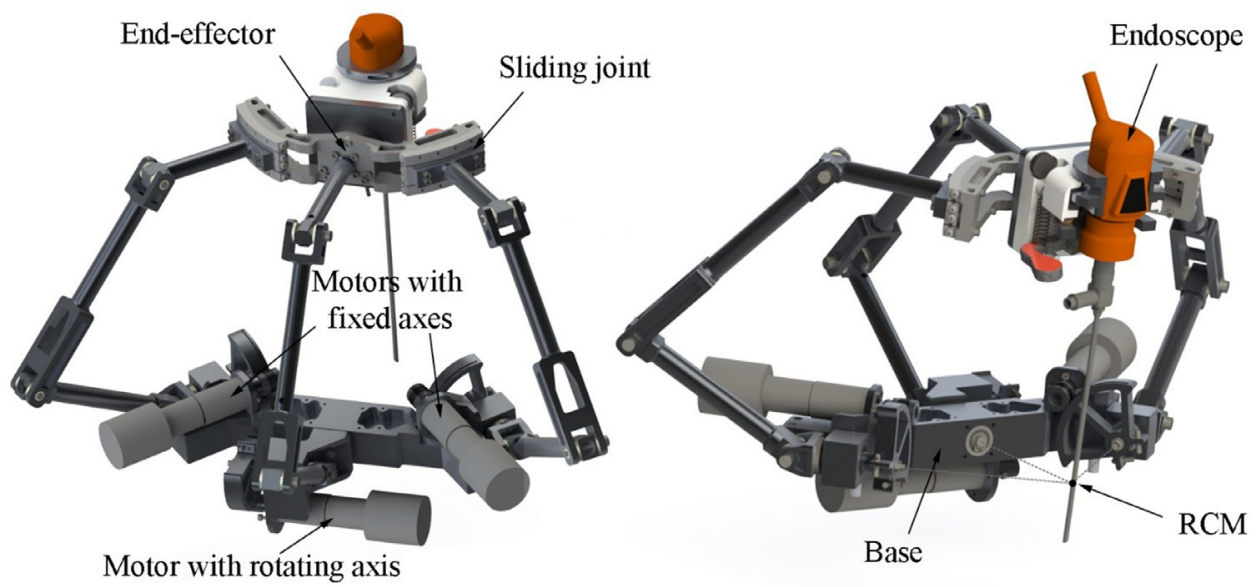

Fig. 11. CAD design of the 2URRR-URR manipulator.

Simscape Multibody Link. As a result of this translation process, the model of the RCM mechanism is generated in Matlab created by Simscape Multibody blocks. Finally, the inverse kinematics equations derived in Section 3.1.2 are included in the simulation model via a Matlab Function block.

The test scenario is developed by using the data acquired from experiments conducted by the neurosurgeons on a cadaver [1]. In this experiment, the neurosurgeons are asked to move the endoscope around the surgical space boundaries for two times while they used the tip of the nose as the pivot point, which is a usual practice during their surgery procedures. During this experiment, rotational motion data is acquired via an inertial measurement unit. This data is later processed and used in the development of the rotational motion profile in the task space for the test scenario. This motion profile is shown in Fig. 9.

During this experiment, the translational motion is not measured but it is obtained by the feedback from the neurosurgeons. The neurosurgeons indicated that once they are inside the surgical area, the endoscope is moved at most $\pm 5 \mathrm{~mm}$. According to this information, the translational motion to be performed during the test is selected to be $d=200+5 \sin (0.2 \pi \mathrm{t})$ $\mathrm{mm}$. The reason to select this frequency of operation is due to the fact that it almost takes $10 \mathrm{~s}$ to complete a full scan of the surgery area boundaries, which can be observed from Fig. 10. In Fig. 10, the joint trajectories are presented which are obtained as a result of the inverse kinematics calculations. The simulation videos taken during this test is available in the additional materials of this article.

\section{CAD design and prototype}

The detailed constructional design of the manipulator is shown in Fig. 11. In the constructional design, the motor axes of the side legs are fixed to the base platform while the motor axis of the middle leg coincides with the second joint axis, hence it is not fixed. It rotates around the fixed $R$ joint axis of the $U$ joint. Also, the motors are connected to the corresponding joints via capstan drives to have a compact design and back-drivability feature. For the side legs, the revolute joints with the endoscope group are constructed by using sliding arc joints. The end-effector of the manipulator consists of the endoscope and a quick-release mechanism. Therefore, the endoscope can be easily detached from the manipulator in case of need during the surgery. While the endoscope is attached to the end-effector, they move as a single body. Lastly, the 


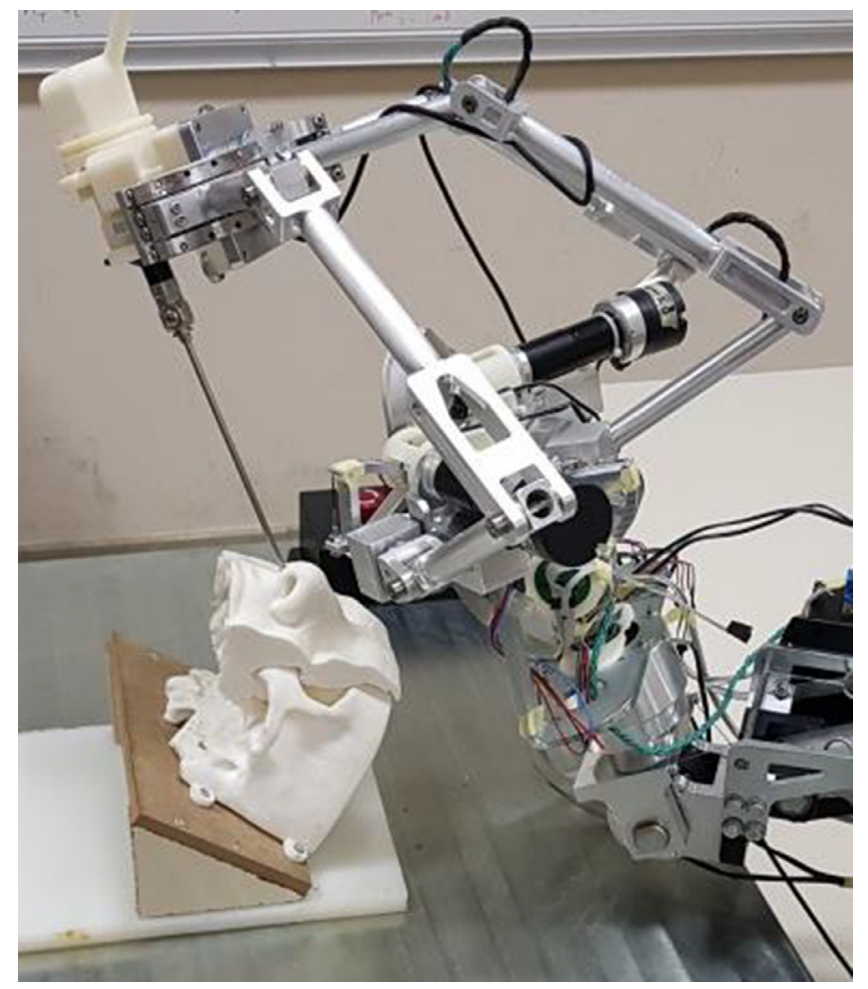

Fig. 12. The prototype of 2URRR-URR manipulator with the mock-up of a transnasal MIS case.

prototype of the manipulator is manufactured and it is presented in Fig. 12 with a mock-up representing the workspace for a transnasal MIS case.

\section{Conclusions}

A 3-dof endoscope holding robot for minimally invasive transnasal surgery applications is presented. This manipulator is designed to be capable of 2R1T motion about/along a RCM. The leg structure is 2URRR-URR. The architecture of the manipulator has been designed to be used as an assistive robot alongside the surgeon in a transnasal surgery by placing the legs on one side of the base platform so that the mechanism minimally occupies the surgeon's workspace. Then, the rotational and translational motions of the end-effector are synthesized by simplifying the spatial architecture of the parallel manipulator to three intersecting planes. After the kinematic analysis is performed, some structural parameters are optimized by using condition number to get optimal transmission characteristics for pitch and yaw motions. Then, slider-crank mechanism design is performed for the middle leg. The dimensional optimization is done using the transmission angle of the mechanism for the desired workspace. The optimized dimensions for the middle leg are also used for the dimensions in side-legs. After the dimensional design is completed, a Matlab simulation is carried out to verify the direct and inverse kinematics formulations. Finally, a CAD model and the manufactured prototype of the designed RCM mechanism are presented.

Most of the parallel-type RCM MIS mechanisms in the literature have axisymmetric designs where the legs are distributed radially symmetric about the end-effector axis. When compared to those mechanisms, the manipulator in this study shows that an asymmetrical design is also possible for parallel-type RCM mechanisms. This asymmetrical design can be beneficial in specific MIS cases where the manipulator is merely assistive whereas the surgeon performs the surgery manually. For a transnasal MIS case, it is shown that the manipulator occupies only one-quarter of the area above the patient's head. Moreover, compared to other parallel mechanisms, the kinematic analysis and design of the manipulator are much simpler such that they can be performed in Microsoft Excel with no need for other complex computational software products and the simple kinematics also helps to develop a simple control algorithm.

\section{Declaration of competing interest}

The authors declare that they have no conflict of interest. 


\section{Acknowledgments}

This study is funded by The Scientific and Technological Research Council of Turkey (grant number 115E726). We would like to thank Prof. Mustafa Berker, Assoc. Prof. Ilkay Işıkay and Dr. Şahin Hanalioğlu for providing valuable inputs for determining the design requirements of the manipulator presented in this article. We also thank Orhan Ayit for helping us with the simulations and Emir Mobedi for the design of the quick-release mechanism for the endoscope.

\section{Supplementary materials}

Supplementary material associated with this article can be found, in the online version, at doi:10.1016/j.mechmachtheory. 2020.104013.

\section{References}

[1] G. Ates, Teleoperation System Design of a Robot Assisted Endoscopic Pituitary Surgery, MSc Thesis, Izmir Institute of Technology, 2018.

[2] A. Bihlmaier, Learning Dynamic Spatial Relations - The Case of a Knowledge-based Endoscopic Camera Guidance Robot, Springer, 2016.

[3] P. Cinquin, J. Troccaz, J. Demongeot, S. Lavallee, G. Champleboux, L. Brunie, F. Leitner, P. Sautot, B. Mazier, A. Perez, M. Djaid, T. Fortin, M. Chenic, A. Chapel, IGOR: image guided operating robot, Innovation et technologie en biologie et médecine 13 (1992) $374-394$.

[4] M.I.C. Dede, Virtual prototyping of robot controllers, International Journal of Design Engineering 3 (3) (2010) 276-288

[5] M.I.C. Dede, G. Kiper, T. Ayav, E. Tatlıcıoğlu, B. Özdemirel, O. Maaroof, G. Ateș, M. Berker, İ. Ișıkay, S. Hanalioğlu, Cerrahın anlık yönlendirilebildiği robot yardımlı endoskop kontrol sistemi mimarisi - NeuRoboScope, in: Proc. Türkiye Robotbilim Konferansı (TORK 2018), İstanbul, 2018, pp. 25-30.

[6] C.H. Kuo, J.S. Dai, Robotics for minimally invasive surgery: a historical review from the perspective of kinematics, in: International Symposium on History of Machines and Mechanisms: Proceedings of HMM 2008, Springer, 2009, pp. 337-354.

[7] C.H. Kuo, J.S. Dai, P. Dasgupta, Kinematic design considerations for minimally invasive surgical robots: an overview, The International Journal of Medical Robotics and Computer Assisted Surgery 8 (2) (2012) 127-145.

[8] Y.S. Kwoh, J. Hou, E.A. Jonckheere, S. Hayati, A robot with improved absolute positioning accuracy for CT guided stereotactic brain surgery, IEEE Transactions on Biomedical Engineering 35 (2) (1988) 153-160.

[9] J. Li, Y. Xing, K. Liang, S. Wang, Kinematic design of a novel spatial remote center-of-motion mechanism for minimally invasive surgical robot, J Med Device 9 (1) (2015) 011003.

[10] J. Li, G. Zhang, A. Müller, S. Wang, A family of remote center of motion mechanisms based on intersecting motion planes, Journal of Mechanical Design 135 (2013) $091009-1$

[11] S. Liu, B. Chen, S. Caro, S. Briot, L. Harewood, C. Chen, A cable linkage with remote centre of motion, Mech Mach Theory 105 (2016) $583-605$.

[12] K. Taniguchi, A. Nishikawa, M. Sekimoto, T. Kobayashi, K. Kazuhara, T. Ichihara, N. Kurashita, S. Takiguchi, Y. Doki, M. Mori, F. Miyazaki, Classification, design and evaluation of endoscope robots, Robot Surgery, InTech, 2010.

[13] R.H. Taylor, A. Menciassi, G. Fichtinger, P. Dario, in: Medical Robotics and Computer-Integrated Surgery, Springer Handbook of Robotics, Springer, 2008, pp. 1199-1222.

[14] A. Yaşır, G. Kiper, Structural synthesis of 2R1T type mechanisms for minimally invasive surgery applications, in: Mechanisms, Transmissions and Applications: Proceedings of the Fourth MeTrApp Conference 2017, Springer, 2018, pp. 31-38. 